\title{
A Time-Dependent Theory of Quantum Resonances ${ }^{\star, \star \star}$
}

\author{
M. Merkli, I. M. Sigal \\ Department of Mathematics, University of Toronto, Toronto, ON, M5S 3G3, Canada. \\ E-mail: merkli@math.toronto.edu; sigal@math.toronto.edu
}

Received: 13 May 1998 / Accepted: 14 September 1998

Abstract: In this paper we further develop a general theory of metastable states resulting from perturbation of unstable eigenvalues. We apply this theory to many-body Schrödinger operators and to the problem of quasiclassical tunneling.

\section{Introduction}

Though the notion of quantum resonance is one of the central notions in physics, the mathematical theory of this phenomenon is still in its early stages. Usually, the resonance is defined in terms of poles of the $S$-matrix or Green's function, bumps in the scattering cross-section, or solutions of the Schrödinger equation with certain boundary conditions at infinity, while its physical picture is that of a metastable state. It is the latter picture that still is very poorly understood and to which our paper aims to make its modest contribution.

We consider in this paper a self-adjoint operator $H_{0}$ on a Hilbert-space $\mathcal{H}$, such that $H_{0}$ has a (possibly degenerate) eigenvalue $E_{0}$ which is embedded in the continuous spectrum of $H_{0}$. We perturb $H_{0}$ by a symmetric operator $W$ such that the operator $H \equiv H_{0}+W$ is self-adjoint, and study the Schrödinger evolution governed by $H$ :

$$
i \partial_{t} \psi=H \psi
$$

We assume that initial conditions are spectrally localized (with respect to $H$ ) in a neighbourhood, $\Delta$, of $E_{0}$ :

$$
\left.\psi_{0} \equiv \psi\right|_{t=0} \in \operatorname{Ran} E_{\Delta}(H) .
$$

Here, $E_{\Delta}(\lambda)$ is the characteristic function of an interval $\Delta$.

\footnotetext{
${ }^{\star}$ Research on this paper is supported by NSERC under Grant NA7901.

$\star \star$ This work is part of the first author's $\mathrm{PhD}$ requirement.
} 
One expects that the eigenvalue $E_{0}$ of $H_{0}$ is unstable under the perturbation $W$, for $W$ sufficiently small. Our goal is to understand how this instability manifests itself on the evolution given by (1.1)-(1.2).

An important additional structure we need is given by a self-adjoint operator $A$ on $\mathcal{H}$, which measures the degree of localization of vectors from $\mathcal{H}$. Namely, vectors in the domain of $\langle A\rangle^{\alpha} \equiv\left(1+|A|^{2}\right)^{\alpha / 2}$ for $\alpha$ sufficiently large are said to be well localized. In other words, by localization of $\psi \in \mathcal{H}$, we mean its localization in the spectral representation of $A$. An orbit $\psi(t) \in \mathcal{H}$ is called dispersive, or locally decaying, if $\left\|\langle A\rangle^{-\alpha} \psi(t)\right\|_{\mathcal{H}}$ vanishes as $t \rightarrow \infty$. An obvious example of an operator $A$ is the coordinate, $x$ (or more precisely, $|x|$ ), if $\mathcal{H}$ is an $L^{2}$-space of functions of $x$. However, sometimes other choices are more convenient, especially the generator of dilation transformations.

Let $P$ be the orthogonal projector of $H_{0}$ onto $\operatorname{Null}\left(H_{0}-E_{0}\right), \bar{P}=11-P$, and let $\bar{H}$ be the reduced Hamiltonian $\bar{H}=\bar{P} H \bar{P}$. We measure the smallness of the perturbation $W$ by the parameter $\kappa \equiv\left\|<A>^{\alpha} W P\right\|$. We show that $\psi$ can be written in the form

$$
\psi(t)=\psi_{\text {res }}(t)+\psi_{\text {disp }}(t),
$$

where $\psi_{\text {res }}(t)=(11+\mathcal{O}(\kappa)) P \psi(t), \psi_{\text {disp }}(t)$ is dispersive, i.e. $\left\|<A>^{-\alpha} \psi_{\text {disp }}(t)\right\|_{\mathcal{H}} \rightarrow 0$ as $t \rightarrow+\infty$, and

$$
P \psi(t)=e^{-i \lambda t} P \psi(0)+\mathcal{O}\left(\kappa^{1-4 \beta}(t+1)^{-\beta}\right),
$$

for some bounded operator $\lambda$ satisfying

$$
\operatorname{Re} \lambda=E_{0} P+\mathcal{O}(\kappa) \text { and } \operatorname{Im} \lambda=-\Gamma+\mathcal{O}\left(\kappa^{3}\right),
$$

where $\Gamma=\pi\left\langle W \bar{P} \delta\left(\bar{H}-E_{0}\right) \bar{P} W\right\rangle_{P}>0$, and we used the notation $\langle A\rangle_{P} \equiv P A P \uparrow$ Ran $P$ for an operator $A$. The delta-function is defined in (A.24).

Equations (1.3)-(1.4) paint the following picture of the resonance behaviour (see Remark 1 of Sect. 2.2 for a technical discussion). A system which is initially localized in a small spectral interval around an unstable eigenvalue radiates energy/probability to infinity, approaches the unstable unperturbed state, stays near it for a period of time of order $1 / \Gamma$, but then eventually loses all the probability to infinity. The decay law (1.5) is given by the celebrated Fermi Golden Rule.

We apply the result above to Schrödinger operators and in particular to $N$-body systems and to the problem of tunneling.

Remarks. 1. Equations (1.3)-(1.5) imply that $H$ has no eigenvalues in the interval $\Delta$, i.e. that the eigenvalue $E_{0}$ of $H_{0}$ is unstable under the perturbation $W$, and that no new eigenvalues emerge. If one is interested in this result alone, a stationary approach would give a simpler proof (cf. [AHS, Sig3] which contain results in this direction).

2 . The perturbation parameter $\kappa$ can be small even when $W$ is large. Exactly this happens in the tunneling resonance problem. In this case $W$ is large but localized in a domain in which $P$ is very small. Our result establishes a relation between the tunneling resonances and the Fermi Golden Rule not observed previously.

3. The bounded operator $\lambda$ describes the resonances of $H$ splitting out of the eigenvalue $E_{0}$ of $H_{0}$ to the order $O\left(\kappa^{3}\right)$. One can extend the proof of (1.3)-(1.5) in order to detect the resonance behaviour and obtain resonances to an arbitrary order. This way, one can replace the condition $\Gamma>0$ by the condition which essentially states that the imaginary 
part of the resonance in question does not vanish at some order (see discussions after Eq. (1.11) and in Sect. 3.1).

4. The assumption $\Gamma>0$ is satisfied generically for one-body Schrödinger operators [AHS]. The same is expected to hold in a much more general context. Under stronger direct assumptions on $H$ this assumption can be essentially removed (see Remark 3 and discussions in Sects. 2.1-2.3 and 3.1).

Strategy. The key tool used in the proof is a linear variant of the Liapunov-Schmidt projection method in the theory of stable and unstable manifolds, or time-dependent variant of the Feshbach method of perturbation theory. Namely we project Eq. (1.1) along the subspaces Ran $P$ and $\operatorname{Ran} \bar{P}$ to obtain the new equations

$$
i \partial_{t} P \psi=\left(E_{0}+\langle W\rangle_{P}\right) P \psi+P W \bar{P} \psi
$$

and

$$
i \partial_{t} \bar{P} \psi=\bar{H} \bar{P} \psi+\bar{P} W P \psi
$$

We will not use the second equation in an essential way; instead, we remark the following: in order to control $\bar{\psi} \equiv \bar{P} \psi$ uniformly in $\kappa$ (or $W$ ), we would like to use a local decay estimate for $e^{-i \bar{H} t} \bar{\psi}(0)$. The latter however does not hold for arbitrary initial conditions $\bar{\psi}_{0} \equiv \bar{\psi}(0)$, but in general only for initial conditions from certain spectral intervals with respect to $\bar{H}$, see Condition (A5) and Sect. 4. Now, even if $\psi_{0}$ is from an appropriate spectral interval for $H$, the initial condition $\bar{\psi}_{0}$ may not be so for $\bar{H}$. To surmount this problem, we observe that since $H$ is very close to $\bar{P} H \bar{P}+P H P$ and since $\psi_{0} \in \operatorname{Ran} E_{\Delta}(H)$, the portion of $\bar{\psi}_{0}$ orthogonal to Ran $g_{\Delta^{\prime}}(\bar{H})$ is very small. Here, $\Delta^{\prime}$ is an interval containing $\Delta$, and $g_{\Delta^{\prime}}(\lambda)$ is a smoothed out characteristic function of the interval $\Delta^{\prime}$ (in particular, $g_{\Delta^{\prime}}(\lambda)=1, \lambda \in \Delta^{\prime}$ ). So we can express $\bar{\psi}$ in terms of the state

$$
\psi_{d} \equiv g_{\Delta^{\prime}}(\bar{H}) \bar{\psi}
$$

and $a$. This ingenious idea was used first by [SW3]. It yields the following representation for $\bar{\psi}$ :

$$
\bar{\psi}=B^{\prime} P \psi+\left(11+B^{\prime}\right) \psi_{d}
$$

where $B^{\prime}=\mathcal{O}(\kappa)$ (i.e. $B^{\prime}$ is a bounded operator with norm $\left\|B^{\prime}\right\| \leq C \kappa$ ). Since $\psi=$ $P \psi_{0}+\bar{\psi}$, the full solution is of the form (1.3) with $\psi_{\text {res }}(t)=B P \psi(t)$ and $\psi_{\text {disp }}=B \psi_{d}$, where $B=11+B^{\prime}=11+\mathcal{O}(\kappa)$.

With Eqs. (1.6) and (1.8)-(1.9) in place, the stage for analysis is set. The component $\psi_{d}$ satisfies the local decay property uniformly in $\kappa$ by Assumption (A4) which is verified for a variety of systems. One important step must be made though, before we embark on estimations of $P \psi(t)$ as a solution to Eq. (1.6): we iterate Eqs. (1.6) and (1.7) (or the resulting equation for $\psi_{d}$ ). This is the only place where Eq. (1.7) is used. The iteration is a rather subtle affair and it takes us to the equation

$$
i \partial_{t} P \psi=\lambda P \psi+f
$$

where $\lambda$ is of the form (1.5) and $f$ satisfies the estimate

$$
r(t) \equiv-i \int_{0}^{t} e^{-i \lambda(t-s)} f(s) d s=O(\kappa), \quad t \geq 0 .
$$


The purpose of this iteration is to pick up the leading imaginary term in the bounded operator $\lambda$ multiplying the function $P \psi(t)$. This leading term is of the second order by Assumption (A5). As a result one needs only one iteration. In general, one should iterate until such a term appears (see discussion in Sect. 3.1). In that case the bounded operator $\lambda$ yields the resonances of $H$ generated by the perturbation of $E_{0}$ to a higher order. Equations (1.10)-(1.11) as well as a priori estimates for the dispersive part $\psi_{d}$ form a foundation of the proof of the result outlined above.

In the analysis above, Ran $P$ plays the role of a stable manifold, so the function $\psi_{\text {res }}(t)$ describes the motion along this manifold and the component $\bar{\psi}$ is expected to decay, at least locally. (This local decay or radiation to infinity differentiates between infinite and finite dimensional dynamical systems.) However, there are two major differences with the standard case. The first one pertains to the pecularity of the resonance problem: The stable manifold is not really stable. It behaves as a stable manifold for long time intervals - the life time of the resonance in question - but eventually it disintegrates itself. The second difference is due to the fact that our problem is linear. This allows us to seek a priori estimates for the dispersive part $\bar{\psi}$, rather than to use the equation for it, Eq. (1.7).

History. Since the early days of quantum physics, the resonance phenomena occupied a central place (see e.g. [BW,KP,S,WW], and [LL] for a textbook discussion). The mathematical theory of resonances could probably be traced to the work of V. Weisskopf and E. Wigner [WW]; in its modern form, it was laid down by B. Simon [Sim1] who used the theory of dilation analytic Hamiltonians due to J. Aguilar and J. M. Combes and E. Balslev and J. M. Combes ([AC, BC]). This approach was further developed in [Sim2, Sig1, Hun2, Ger, HeSj, HM]. Details and extensive references can be found in [HiSig]. It requires the potential in question to have some analyticity properties at least in a neighbourhood of infinity. A theory dispensing with the latter condition was initiated in [Ort].

So far the theory developed was a stationary one, despite the fact that the physical picture (but not the physical definitions!) was that of a metastable state. The timedependent theory was initiated in works of E. Skibsted and W. Hunziker ([Sk1,2, Hu2]), and a space-time and the phase-space-time and variational analysis was given in [GS] and $[\mathrm{PF}]$, respectively. A new powerful approach was suggested by A. Soffer and M. Weinstein [SW3], who also obtained a rather detailed space-time description of evolution of metastable (resonance) states in the one-body Schrödinger case and for a Schrödinger particle coupled to a massive quantum field. Our paper generalizes the result of [SW3] to many-body Schrödinger operators (and degenerate eigenvalues). Besides we also treat the case of quasiclassical resonances, not considered in [SW3]. Though our approach follows the same general line as that of [SW3], we had to introduce some essential changes right at the beginning in order to make it applicable to a considerably wider class of systems.

The approach outlined above was introduced in [SW1] (see also [SW2] and [BP]). The latter work was further improved and coached in terms of the stable-unstable manifold theory in [PW]. This approach is in fact what is known in physics as the (Feshbach) projection method (with the projection operator $P$ ). It is usually applied to the stationary Schrödinger equation $(H-E) \psi=0$, while we apply it to the Schrödinger equation $\left(H-i \partial_{t}\right) \psi=0$. It serves also as a starting point to a renormalization group construction in a recent work [BFS1-3].

Notation. We use the following notation besides the one introduced above: $E_{\Delta}(\lambda)$ denotes the characteristic function of an interval $\Delta$, so that $E_{\Delta}(H)$ is the spectral projector of 
$H$ corresponding to the interval $\Delta$. The length of $\Delta$ is written as $|\Delta|$. A smoothened characteristic function of an open bounded interval $\Delta \subset \mathbb{R}$ is denoted as $g_{\Delta}$, i.e. $g_{\Delta} \in$ $C_{0}^{\infty}\left(\Delta_{1}\right)$, where $\Delta_{1}$ is a slightly bigger interval than $\Delta$, and $g_{\Delta}(\lambda)=1$ on $\Delta$. We set $\bar{g}_{\Delta} \equiv 1-g_{\Delta}$.

The norm in the Hilbertspace $\mathcal{H}$ is denoted as $\|\cdot\|_{\mathcal{H}}$, and $\langle\cdot, \cdot\rangle$ is its inner product. The expectation value of an operator $B$ in the state $\psi$ is written as $\langle B\rangle_{\psi} \equiv\langle\psi, B \psi\rangle$. Moreover, $\langle B\rangle_{P}$ stands for the operator $P B P \uparrow \operatorname{Ran} P$ on the space $\operatorname{Ran} P$.

The domain of an (unbounded) operator $A$ on $\mathcal{H}$ is written as $\mathcal{D}(A)$. For $t \in \mathbb{R}$, let $<t>\equiv\left(1+t^{2}\right)^{1 / 2}$, and $\left\langle A>=\left(1+A^{*} A\right)^{1 / 2}\right.$, where $A^{*}$ denotes the adjoint. We also let $\operatorname{Re} A=\left(A+A^{*}\right) / 2$, and $\operatorname{Im} A=\left(A-A^{*}\right) / 2$.

For a self-adjoint operator $H$, we set $\delta(H-\lambda)=\frac{1}{\pi} \operatorname{Im}(H-\lambda-i 0)^{-1}$, and P.V. $(H-$ $\lambda)^{-1}=\operatorname{Re}(H-\lambda-i 0)^{-1}$, where the r.h.s. are assumed to be defined between appropriate Banach spaces.

Let $\mathcal{L}(\mathcal{H})$ be the space of bounded linear operators on $\mathcal{H}$ with the standard norm $\|\cdot\|$. For a family $\left\{B_{s}\right.$ rbrace of operators in $\mathcal{L}(\mathcal{H})$ depending on the parameter $s \geq 0$, we write $B_{s}=\mathcal{O}(s)$ if $\left\|B_{s}\right\| \leq C s$, where $C$ is a constant independent of $s$. For a complexvalued function $f(s), f(s)=O(s)$ means $|f(s)| \leq C s$ and for $\phi_{s} \in \mathcal{H}, \phi_{s}=O(s)$ means $\left\|\phi_{s}\right\|_{\mathcal{H}}=O(s)$.

Finally, for notational convenience, $C$ will denote a generic strictly positive constant whose value can vary from expression to expression ( $C$ is allowed to depend on $\alpha, \beta,|\Delta|$, see below, but not on $\kappa$ or $t$ ).

\section{Main Results}

2.1. Assumptions. We will work in the setting presented above, and where $\operatorname{dim} \operatorname{Ran} P \leq$ $\infty$. The operators $H_{0}$ and $H$ are assumed to be self-adjoint on the same domain, and $W$ to be symmetric. We also assume that there is a self-adjoint operator $A$ and a number $\alpha>2$ such that:

(A1) $\left\|<A>^{\alpha} P\right\|<\infty$,

(A2) the perturbation $W$ satisfies $\kappa \equiv\left\|<A>^{\alpha} W P\right\|<\infty$,

(A3) the multi-commutators $a d_{A}^{(k)}(H)$ are $H$-bounded, uniformly in $W, k=1, \ldots, n$, where $n>\alpha+1$

(A4) the following local decay estimate holds for all $\phi \in \mathcal{D}\left(<A>^{\alpha}\right)$ and $t \geq 0$ :

$$
\left\|<A>^{-\alpha} e^{-i \bar{H} t} g_{\Delta^{\prime}}(\bar{H}) \bar{P} \phi\right\|_{\mathcal{H}} \leq C<t>^{-\alpha}\left\|<A>^{\alpha} \bar{P} \phi\right\|_{\mathcal{H}}
$$

for some constant $C>0$ independent of $W$,

(A5) the Fermi Golden Rule condition holds for $E_{0}$ in the sense that there is a $C_{0}>0$ such that the bounded, nonnegative operator

$$
\Gamma \equiv \pi\left\langle W \delta\left(\bar{H}-E_{0}\right) \bar{P} W\right\rangle_{P}
$$

satisfies:

$$
\Gamma \geq C_{0} \kappa^{2}
$$

Remarks. 1. (A1)-(A3) are easily verified in the applications (see Sect. 2.3).

2. The uniformity clause in (A3) and (A4) is a restriction on the class of $W$ 's allowed for a given $H_{0}$. 
3. In Sect. 4, we derive Condition (A4) from the Mourre estimate. It is much easier to check the latter, as we demonstrate in examples below.

4. It is shown in the appendix that $\Gamma$ is well defined and satisfies $\|\Gamma\| \leq C \kappa^{2}$ (see the remark after (A.24)). Hence (A5) gives

$$
C_{0} \kappa^{2} \leq\|\Gamma\| \leq C \kappa^{2}
$$

5. If the Mourre estimate holds then one can show (see e.g. [Sig2]) that $\Gamma=\gamma \kappa^{2}+\mathcal{O}\left(\kappa^{7 / 3}\right)$, where $\gamma=\pi\left\langle U \delta\left(\bar{H}_{0}-E_{0}\right) \bar{P} U\right\rangle_{P}$, and $U=W / \kappa$.

6. Condition (A5), or its more precise form given in Remark 5, is conjectured to hold generically. For a more detailed discussion in the case of Schrödinger operators see Paragraph 2.3. In fact, Condition (A5) can be removed at the expense of requiring a larger $\alpha$ in Conditions (A1)-(A4) and a lengthier proof (see discussion in Sect. 3.1).

7. The smoothness Condition (A3) can be thought of as a $C^{n}(\mathbb{R})$ property of the family $H(\theta)=U(\theta)^{-1} H U(\theta)$, where $U(\theta)=\exp (i \theta A)$, the dilation group.

2.2. Abstract result. We present our main result in the setting of a general Hilbert-space (in Theorem 2.1). We treat the case of Schrödinger operators in Sect. 2.3.

Theorem 2.1. Let $\alpha>2$ and $0 \leq \beta<\min \{1 / 2, \alpha-2\}$ and let $\psi$ be the solution of $i \partial_{t} \psi=H \psi$, with initial condition $\psi_{0} \in \operatorname{Ran} E_{\Delta}(H) \cap \mathcal{D}\left(<A>^{\alpha}\right)$. Then there exists $a$ constant $\kappa_{0}$ (depending on $\alpha, \beta$ and $\left.|\Delta|\right)$ such that for $\kappa<\kappa_{0}$ we have the expansion:

$$
\psi(t)=(l l+\mathcal{O}(\kappa)) P \psi(t)+\psi_{\text {disp }}(t),
$$

where $\psi_{\text {disp }}(t)$ is a dispersive wave satisfying for $t \geq 0$ :

$$
\left\|<A>^{-\alpha} \psi_{\text {disp }}(t)\right\|_{\mathcal{H}} \leq C\left(\left\|<A>^{\alpha} \bar{P} \psi_{0}\right\|_{\mathcal{H}}<t>^{-\alpha}+\kappa^{1-2 \beta}<t>^{-\beta}\right),
$$

and $P \psi(t)$ satisfies for $t \geq 0$ :

$$
P \psi(t)=e^{-i \lambda t} P \psi_{0}+\mathcal{O}\left(\kappa^{1-4 \beta}<t>^{-\beta}\right) .
$$

Here $\lambda$ is a bounded linear operator on Ran $P$ satisfying

$$
\operatorname{Re} \lambda=E_{0} P+P W P-P W\left(\text { P.V. }\left(\bar{H}-E_{0}\right)^{-1}\right) \bar{P} W P+\mathcal{O}\left(\kappa^{3}\right),
$$

and

$$
\operatorname{Im} \lambda=-\Gamma+\mathcal{O}\left(\kappa^{3}\right) .
$$

The terms on the r.h.s. of (2.7) and (2.8) are well defined.

Corollary 2.2. Under the assumptions of Theorem 2.1, $H$ has no eigenvalues in the interval $\Delta$.

Remarks. 1. Equations (2.4)-(2.8) imply that though any orbit $\psi(t)$ starting at $\psi_{0} \in$ Ran $E_{\Delta}(H)$ is dispersive (i.e. locally decaying), for $\left\|\bar{P} \psi_{0}\right\|_{\mathcal{H}}$ small and for any $R>0$, the state $\chi_{|A| \leq R} \psi(t)$ is close to the "stationary" state $\chi_{|A| \leq R} e^{-i \lambda t} P \psi_{0}$ in a time interval of the order $\frac{1}{\kappa} \ln \left(\kappa^{-1}\right)$.

2. Our analysis yields a more detailed information about $P \psi(t)$ and $\psi_{\text {disp }}(t)$ than the one given in Theorem 2.1. The dispersive wave satisfies 


$$
\begin{aligned}
\left\|<A>^{-\alpha} \psi_{\text {disp }}(t)\right\|_{\mathcal{H}} \leq & C\left\|<A>^{\alpha} \bar{P} \psi_{0}\right\|_{\mathcal{H}}\left(<t>^{-\alpha}+<t>^{-\beta} \kappa^{2-2 \beta}\right) \\
& +C\left\|P \psi_{0}\right\|_{\mathcal{H}}<t>^{-\beta} \kappa^{1-2 \beta} .
\end{aligned}
$$

Set $P \psi(t)=e^{-i \lambda t} P \psi(0)+r(t)$. Then we have in particular

$$
\|r(t)\|_{\mathcal{H}} \leq C\left(\left\|<A>^{\alpha} \bar{P} \psi_{0}\right\|_{\mathcal{H}} \kappa^{1-2 \beta}+\left\|P \psi_{0}\right\|_{\mathcal{H}} \kappa^{1-4 \beta}\right)<t>^{-\beta} .
$$

3. There are various versions of Theorem 2.1 in which the condition $\psi_{0} \in \operatorname{Ran} E_{\Delta}(H) \cap$ $\mathcal{D}\left(\langle A\rangle^{\alpha}\right)$ is either relaxed or modified (e.g. to $\psi_{0} \in \operatorname{Ran} P$ ).

2.3. Schrödinger operators. In this section, we choose our Hilbert-space to be $\mathcal{H}=$ $L^{2}(X) \equiv L^{2}$ (with norm $\|\cdot\|_{2}$ ), where $X$ is a finite dimensional inner product space, the configuration space of a system in question. The Hamiltonian is given by

$$
H_{0}=-\Delta+V
$$

where $\Delta$ is the Laplacian on $X$, and $V$ is a real function on $X$ called the potential. Our choice of the operator $A$ is the dilation generator

$$
A=\frac{1}{2}(x \cdot p+p \cdot x)
$$

where $p=-i \nabla$, and the dot product is a coupling of $X$ and $X^{\prime}$. We assume here that $W=\kappa^{\prime} U$, where $\kappa^{\prime}$ is a real number, and $U: X \rightarrow \mathbb{R}$, $\operatorname{dim} \operatorname{Ran} P<\infty$. Setting $\kappa \equiv\left\|<A>^{\alpha} W P\right\|$ as before, we have $\kappa=\kappa^{\prime}\left\|<A>^{\alpha} U P\right\|$, and $\kappa \rightarrow 0$ is equivalent to $\kappa^{\prime} \rightarrow 0$, provided $\left\|<A>^{\alpha} U P\right\|<\infty$ (see (2.11)). Making the perturbation small means here to make $\kappa^{\prime}$ small. We assume that for some $n>\alpha+1$ :

(S1) $V, U \in C^{n}$, with bounded derivatives,

(S2) (i) $(x \cdot \nabla)^{k} V$ is $H$-bounded, $0 \leq k \leq n$,

(ii) $(x \cdot \nabla)^{k} U$ is $H$-bounded, $0 \leq k \leq n$,

(S3) there exists a neighbourhood $\Delta_{1}$ of $E_{0}$ such that

$$
E_{\Delta_{1}}\left(H_{0}\right) i\left[H_{0}, A\right] E_{\Delta_{1}}\left(H_{0}\right) \geq \theta_{1} E_{\Delta_{1}}\left(H_{0}\right)+K,
$$

where $\theta_{1}>0$ and $K$ is a compact operator on $L^{2}$,

(S4) the Fermi Golden Rule Condition holds in the sense that

$$
\Gamma=\pi\left(\kappa^{\prime}\right)^{2}\left\langle U \delta\left(\bar{H}_{0}-E_{0}\right) \bar{P} U\right\rangle_{P}>0
$$

( $\Gamma$ is positive definite).

Conditions (S2) with $k=0,1$ and (S3) imply that the projection $P$ satisfies the following two estimates:

$$
\left\|<A>^{\alpha} U P\right\|<\infty
$$

and for any multi-indices $\mathbf{m}_{1}$ and $\mathbf{m}_{2}$ with $\left|\mathbf{m}_{1,2}\right| \leq n$ :

$$
\left\|x^{\mathbf{m}_{1}} p^{\mathbf{m}_{2}} P\right\|<\infty .
$$

Indeed, proceeding as in [HS1,CFKS], one can show that there is a $\delta>0$ such that $\left\|e^{\delta|x|} P\right\|<\infty$. Using then $\left(p^{2}+V\right) P=E_{0} P$, and Condition (S1), it is easily shown that ||$e^{\delta|x|} p^{\mathbf{m}} P \|<\infty$, for $|\mathbf{m}| \leq n$, from which (2.11) and (2.12) follow. 
Remark. In the one-body case (see e.g. the problem of tunneling in Sect. 2.3.2), Condition (S4) was shown to hold generically at least for $E_{0}$ simple ([AHS], for more details see below). The latter is still a conjecture in the many-body case. The only general results in that case are those of [AHS]. Translated into our context they state, under some additional conditions, that $\delta\left(\bar{H}_{0}-E_{0}\right) \neq 0$ and that, given a many-body Hamiltonian $H_{0}$ as above and an open set $\Omega \subset X$ (for the definition of $X$ see Sect. 2.3.1) there is a real potential $G \in C_{0}^{\infty}(\Omega)$, s.t. $\left\langle G \delta\left(\bar{H}-E_{0}\right) G\right\rangle_{P}>0$ at least for $E_{0}$ simple.

Theorem 2.3. Theorem 2.1 holds for the Schrödinger operator (2.10) if we replace Conditions (A1)-(A5) by (S1)-(S4).

The proof of Theorem 2.3 is given in Sect. 5. It consists in showing that the Conditions (S) imply the Conditions (A) in the case of Schrödinger operators. The essential part is to show that starting from the Mourre estimate (S3) for $H_{0}$, we get a strong Mourre estimate for the reduced Hamiltonian $\bar{H}$, see Theorem 4.1, and therefore the local decay estimate (A4) for $\bar{H}$.

2.3.1. $N$-body systems. For $N$-body systems in the physical space $\mathbb{R}^{d}$, the configuration space is

$$
X=\left\{x=\left(x_{1}, \ldots, x_{N}\right) \in \mathbb{R}^{d N} \mid \sum m_{i} x_{i}=0\right\} .
$$

Here $m_{i}$ is the mass of particle $i$. The Hamiltonian $H_{0}$ is a Schrödinger operator (2.10) with $V_{0}$, an $N$-body potential:

$$
V_{0}(x)=\sum_{i<j} V_{i j}\left(x_{i}-x_{j}\right),
$$

where the two-body potentials $V_{i j}: \mathbb{R}^{d} \rightarrow \mathbb{R}$ vanish at infinity. In terms of the two-body potentials, (S2)(i) means that $(x \cdot \nabla)^{k} V_{i j}$ is $\Delta$-bounded, $0 \leq k \leq n$. For example, we can take $(x \cdot \nabla)^{k} V_{i j}$ to be Kato-potentials on $\mathbb{R}^{d}, 0 \leq k \leq n$. Similarly, (S2)(ii) is satisfied if $(x \cdot \nabla)^{k} U$ are Kato-potentials on $\mathbb{R}^{d N}$, for $0 \leq k \leq n$.

If $E_{0}$ is separated from the thresholds of $H_{0}$, then $E_{0}$ is finitely degenerate, $\operatorname{dim} \operatorname{Ran} P<\infty$ (see [HS1,CFKS]), and the Mourre estimate holds for $H_{0}$ and $A$ if $\Delta_{1}$ is sufficiently small. Thus we obtained

Corollary 2.4. The conclusion of Theorem 2.1 holds under the assumptions on the potentials mentioned above, provided that $E_{0}$ is separated from the thresholds of $H_{0}$, and if the Fermi Golden Rule Condition holds.

2.3.2. Tunneling in quasiclassical approximation. We consider the following initial value problem on $L^{2}\left(\mathbb{R}^{d}\right)$ :

$$
\begin{aligned}
i \hbar \partial_{t} \psi & =H \psi \\
\left.\psi\right|_{t=0} \equiv \psi_{0} & \in \operatorname{Ran} E_{\hbar \Delta}(H),
\end{aligned}
$$

where $\hbar>0$ is considered to be a small parameter, and $\Delta$ is an interval to be specified, lying at the bottom of the continuous spectrum of $H$, and $\hbar \Delta \equiv\{\hbar E \mid E \in \Delta\}$. Here the Schrödinger operator $H$ is given by

$$
H=p^{2}+V, \quad p=-i \hbar \nabla,
$$

and $V$ is a volcano-shaped bounded potential with a local minimum at the origin, defined in the Conditions (T) below. We define a reference potential $V_{0}$, such that 
(i) $V_{0}$ is $C^{n}\left(\mathbb{R}^{d}\right)$, and such that $(x \cdot \nabla)^{k} V_{0}$ is bounded, $0 \leq k \leq n$,

(ii) $V_{0}(x)=V(x)$ in a neighbourhood $\mathcal{N}$ of the origin, and $\inf _{x \in \mathbb{R}^{d} \backslash \mathcal{N}} V_{0}(x)>V(0)$.

We set

$$
H_{0}=p^{2}+V_{0},
$$

and therefore $H=H_{0}+W$ with $W=V-V_{0}$. For $\hbar$ small enough, $H_{0}$ has a unique (normalized) ground-state $\varphi_{0}$ with energy $E_{0}$ separated from the continuous spectrum of $H_{0}$. Let $A \equiv(x \cdot p+p \cdot x) /(2 \hbar)$ and $\kappa \equiv\left\|<A>^{\alpha} W \varphi_{0}\right\|_{2}$.

We give now conditions on the potential $V$, and state then the result of this section, Theorem 2.5.

(T1) $V \geq 0, V \in C^{n}\left(\mathbb{R}^{d}\right)$, and $(x \cdot \nabla)^{k} V$ is bounded, $0 \leq k \leq n$,

(T2) $V$ has a non-degenerate local minimum at $x=0$ and vanishes as $|x| \rightarrow \infty$,

(T3) there is a $\delta>0$ such that $\Omega_{\delta} \equiv\left\{x \in \mathbb{R}^{d} \mid V(x)<V(0)+\delta\right\}$ has a connected component $\Omega_{\delta}^{\text {ext }}$ containing $\infty$, on which $V(x)$ is non-trapping: $\forall x \in \Omega_{\delta}^{e x t}$,

$$
2(V(0)-V(x))-x \cdot \nabla V(x) \geq \theta, \quad \text { for some } \theta>0,
$$

(T4) the Fermi Golden Rule Condition holds: $\Gamma \equiv \pi\left\langle\delta\left(\bar{H}-E_{0}\right)\right\rangle_{\bar{P} W \varphi_{0}}$ satisfies $\Gamma \geq$ $C_{0} \hbar^{p} \kappa^{2}$, for some $p \geq 0$.

Remarks. 1. By non-degeneracy of the local minimum in (T2), we mean that the Hessian of $V$ at 0 is strictly positive definite.

2. From the harmonic approximation (see e.g. [HiSig], Chapter 11), it follows that $E_{0}=$ $V(0)+O(\hbar)$. Hence for small $\hbar$, Condition (ii) implies that the classically allowed region $\left\{x \in \mathbb{R}^{d} \mid V_{0}(x) \leq E_{0}\right\}$ is compact. This implies that $\varphi_{0}$ is localized in $x$ exponentially around the origin: $\forall \epsilon, \epsilon^{\prime}>0, \exists C_{\epsilon, \epsilon^{\prime}}$ (independent of $\hbar$ ) such that

$$
\left\|e^{(1-\epsilon) \rho_{E_{0}} / \hbar} \varphi_{0}\right\|_{2} \leq C_{\epsilon, \epsilon^{\prime}} e^{\epsilon^{\prime} / \hbar} .
$$

Here, $\rho_{E_{0}}(x)$ is the geodesic distance between $x$ and 0 , measured in the Agmon metric corresponding to the energy $E_{0}$. The proof of (2.18) is easily obtained e.g. from [HiSig], Theorem 3.4.

3 . Since $E_{0}=V(0)+O(\hbar)$, then for small $\hbar, W$ is supported in the classically forbidden region $\left\{x \in \mathbb{R}^{d} \mid V_{0}(x)>E_{0}\right\}$. The exponential decay (in $x$ ) of $\varphi_{0}$ implies then that $\kappa=\left\|<A>^{\alpha} W \varphi_{0}\right\|_{2}=O\left(e^{-\eta / \hbar}\right)$, for some $\eta>0$.

Theorem 2.5. Assume Conditions (T1)-(T4) hold, and let $0 \leq \beta<\min \{\alpha-2,1 / 2\}$. Then there is a $\hbar_{0}>0$ (dependent on $\alpha, \beta, n,|\Delta|, p$ ) such that for $\hbar<\hbar_{0}$, the solution to (2.13)-(2.14) has the expansion

$$
\psi(t)=a(t) \varphi_{1}+\psi_{d i s p}(t)
$$

where $\varphi_{1}=\varphi_{0}+O(\kappa / \hbar)$, and $\psi_{\text {disp }}(t)$ is a dispersive wave satisfying for $t \geq 0$ :

$$
\begin{aligned}
\left\|<A>^{-\alpha} \psi_{\text {disp }}(t)\right\|_{2}= & \left\|<A>^{\alpha} \bar{P} \psi_{0}\right\|_{2} O\left(<t>^{-\alpha} \hbar^{-q}\right) \\
& +O\left(<t>^{-\beta} \hbar^{-q} \kappa^{1-2 \beta}\right),
\end{aligned}
$$

with q, a positive integer depending on $\alpha, \beta, n, p$. Moreover, $a(t)=\left\langle\varphi_{0}, \psi(t)\right\rangle$ satisfies for $t \geq 0$ : 


$$
a(t)=e^{-i \lambda t / \hbar} a(0)+O\left(<t>^{-\beta} \hbar^{-q} \kappa^{1-4 \beta}\right),
$$

with

$$
\begin{aligned}
& \operatorname{Re} \lambda=E_{0}+\langle W\rangle_{\varphi_{0}}-\left\langle P . V .\left(\bar{H}-E_{0}\right)^{-1}\right\rangle_{\bar{P} W \varphi_{0}}+O\left(\hbar^{-2} \kappa^{3}\right), \\
& \operatorname{Im} \lambda=-\pi\left\langle\delta\left(\bar{H}-E_{0}\right)\right\rangle_{\bar{P} W \varphi_{0}}+O\left(\hbar^{-2} \kappa^{3}\right) .
\end{aligned}
$$

We prove Theorem 2.5 in Sect. 6. A general discussion of the quasiclassical Schrödinger problem and extensive references can be found in [HiSig].

\section{Proof of Theorem 2.1}

The proof of Theorem 2.1 consists of two main steps. In a first step, we establish differential equations for $P \psi$ and $\psi_{d}(t)$ (the latter function is defined in (1.8)), write the corresponding integral equations, and bring these equations to a convenient form. We do this in Sect. 3.1. In the second step we use these equations in order to prove the desired estimates on $P \psi(t)$ and $\psi_{d}(t)$. This is done in Sect. 3.2. Theorem 2.1 is then derived by observing that

$$
\psi(t)=B\left(P \psi(t)+\psi_{d}(t)\right),
$$

where the operator $B$ satisfies $B=11+\mathcal{O}(\kappa)$.

3.1. Differential equations for $P \psi(t)$ and $\psi_{\text {disp }}(t)$. In this section, we establish the coupled differential equations for $P \psi$ and $\psi_{d}$ and iterate them in a suitable manner. The main result here is Eq. (3.13) together with the set of equations (3.14). Projecting (1.1) onto Ran $P$ and Ran $\bar{P}$ yields

$$
\begin{aligned}
i \partial_{t} P \psi & =\left(E_{0}+P W P\right) P \psi+P W \bar{P} \psi, \\
i \partial_{t} \bar{P} \psi & =\bar{H} \bar{P} \psi+\bar{P} W P \psi .
\end{aligned}
$$

Recall that $\psi_{d}=P_{d} \psi$, where $P_{d}=g_{\Delta^{\prime}}(\bar{H}) \bar{P}$. To pass from $\bar{P} \psi$ to $\psi_{d}$, we multiply both sides of (3.3) by $g_{\Delta^{\prime}}(\bar{H})$ and get

$$
i \partial_{t} \psi_{d}=\bar{H} \psi_{d}+P_{d} W P \psi
$$

Now we express $\bar{P} \psi$ in (3.2) in terms of $P \psi$ and $\psi_{d}$. Notice that $\psi_{0} \in \operatorname{Ran} E_{\Delta}(H)$, so that $\psi_{0}=g_{\Delta}(H) \psi_{0}$. Hence $\psi=g_{\Delta}(H) \psi$ and therefore $\bar{P} \psi=\bar{P} g_{\Delta}(H) \psi$. Introducing $11=g_{\Delta^{\prime}}(\bar{H})+\bar{g}_{\Delta^{\prime}}(\bar{H})$ into the last equation yields

$$
\begin{aligned}
\bar{P} \psi & =\left(g_{\Delta^{\prime}}(\bar{H})+\bar{g}_{\Delta^{\prime}}(\bar{H})\right) \bar{P} g_{\Delta}(H) \psi \\
& =\bar{g}_{\Delta^{\prime}}(\bar{H}) \bar{P} g_{\Delta}(H) \psi+g_{\Delta^{\prime}}(\bar{H}) \bar{P} \psi \\
& =\bar{g}_{\Delta^{\prime}}(\bar{H}) \bar{P} g_{\Delta}(H) \bar{P} \psi+\bar{g}_{\Delta^{\prime}}(\bar{H}) \bar{P} g_{\Delta}(H) P \psi+P_{d} \psi
\end{aligned}
$$

Hence $\left(11-\bar{g}_{\Delta^{\prime}}(\bar{H}) \bar{P} g_{\Delta}(H)\right) \bar{P} \psi=\bar{g}_{\Delta^{\prime}}(\bar{H}) \bar{P} g_{\Delta}(H) P \psi+\psi_{d}$. The following proposition is proven in the appendix:

Proposition 3.1. $\bar{g}_{\Delta^{\prime}}(\bar{H}) \bar{P} g_{\Delta}(H)=\mathcal{O}(\kappa)$ and consequently, for small $\kappa, B \equiv(11-$ $\left.\bar{g}_{\Delta^{\prime}}(\bar{H}) \bar{P} g_{\Delta}(H)\right)^{-1}$ exists as a bounded linear operator on $\mathcal{H}$, and $\|B\| \leq C$ (uniformly in $\kappa$ for small $\kappa)$. 
We have therefore

$$
\bar{P} \psi=B^{\prime} P \psi+B \psi_{d}
$$

where $B^{\prime}=B-11$, and $B$ is defined in Proposition 3.1 above. Remark that $B=11+\mathcal{O}(\kappa)$, and $B^{\prime}=\mathcal{O}(\kappa)$. With expression (3.5) for $\bar{P} \psi$, we get

$$
\psi(t)=B P \psi(t)+\psi_{\text {disp }}(t),
$$

where we defined

$$
\psi_{\text {disp }}(t)=B \psi_{d}(t) .
$$

Equation (3.6) shows that $\psi$ is of the form (2.4).

From (3.2) and (3.5), we have the following equation of motion for $P \psi$ :

$$
i \partial_{t} P \psi=\lambda_{1} P \psi+P W B \psi_{d},
$$

where $\lambda_{1}$ is a bounded linear operator on $\operatorname{Ran} P$ :

$$
\lambda_{1}=E_{0}+P W B P .
$$

Next, we rewrite Eq. (3.4) in the integral form

$$
\psi_{d}(t)=e^{-i \bar{H} t} \psi_{d}(0)-i \int_{0}^{t} e^{-i \bar{H}(t-s)} P_{d} W P \psi(s) d s .
$$

The last term can be transformed as follows: pick $z \in \mathbb{C}^{+}$and integrate by parts in the following way:

$$
\begin{aligned}
- & -\int_{0}^{t} e^{-i \bar{H}(t-s)} P_{d} W P \psi(s) d s \\
= & -i e^{-i \bar{H} t} \int_{0}^{t} e^{i(\bar{H}-z) s} e^{i z s} P_{d} W P \psi(s) d s \\
= & -(\bar{H}-z)^{-1} P_{d} W P \psi(t)+e^{-i \bar{H} t}(\bar{H}-z)^{-1} P_{d} W P \psi(0) \\
& +\int_{0}^{t} e^{-i \bar{H}(t-s)}(\bar{H}-z)^{-1} P_{d} W\left[i z P \psi(s)+\left(\partial_{s} P \psi\right)(s)\right] d s .
\end{aligned}
$$

In order to make the last term on the r.h.s. of (3.11) small, we want to take $z$ to the real axis. Such a procedure is justified in the first two statements of the proposition to follow. The third statement of this proposition shows why $z$ must approach the real axis from above for $t>0$ (the outgoing condition).

Proposition 3.2. For $\omega \in \mathbb{R}$ and $\phi \in \mathcal{H} \cap \mathcal{D}\left(<A>^{\alpha}\right)$, we have:

(i) $\forall t \geq 0:<A>^{-\alpha} e^{-i \bar{H} t}(\bar{H}-\omega-i \epsilon)^{-1} P_{d} \phi$ converges in $\mathcal{H}$ as $\epsilon \downarrow 0$. The limit is denoted as $\langle A\rangle^{-\alpha} e^{-i \bar{H} t}(\bar{H}-\omega-i 0)^{-1} P_{d} \phi$.

(ii) The convergence in (i) is uniform in $t \in \mathbb{R}_{+}$, and therefore $t \mapsto\langle A\rangle^{-\alpha} e^{-i \bar{H} t}(\bar{H}-$ $\omega-i 0)^{-1} P_{d} \phi$ is continuous as a map from $\mathbb{R}_{+}$to $\mathcal{H}$.

(iii) $\left\|<A>^{-\alpha} e^{-i \bar{H} t}(\bar{H}-\omega-i 0)^{-1} P_{d} \phi\right\|_{\mathcal{H}} \leq C(t+1)^{-\alpha+1}\left\|<A>^{\alpha} \bar{P} \phi\right\|_{\mathcal{H}}, \forall t \geq 0$. 
This proposition is proven in the appendix.

Notice that as $\operatorname{Im} z \rightarrow 0$, the individual terms in (3.11) do not converge in $\mathcal{H}$. However, setting $\omega=\operatorname{Re} z$, and using our assumption that $\left\langle A>^{\alpha} W P\right.$ is a bounded operator (see (A2)) and Proposition 3.2, we get for $P W B$ acting on the second term on the r.h.s. of (3.10):

$$
\begin{aligned}
& -i \int_{0}^{t} P W B e^{-i \bar{H}(t-s)} P_{d} W P \psi(s) d s \\
& =-i \int_{0}^{t} P W B^{\prime} e^{-i \bar{H}(t-s)} P_{d} W P \psi(s) d s \\
& \quad-P W(\bar{H}-\omega-i 0)^{-1} P_{d} W P \psi(t)+P W e^{-i \bar{H} t}(\bar{H}-\omega-i 0)^{-1} P_{d} W P \psi(0) \\
& \quad+i \int_{0}^{t} P W e^{-i \bar{H}(t-s)}(\bar{H}-\omega-i 0)^{-1} P_{d} W\left(\omega-\lambda_{1}\right) P \psi(s) d s \\
& \quad-i \int_{0}^{t} P W e^{-i \bar{H}(t-s)}(\bar{H}-\omega-i 0)^{-1} P_{d} W P W B \psi_{d}(s) d s,
\end{aligned}
$$

where the term $P W(\bar{H}-\omega-i 0)^{-1} P_{d} W P \psi(t)$ and similar ones are well defined. To get the last two terms, we replaced $\partial_{t} P \psi$ in (3.11) using (3.8). Expression (3.12) contains the term of order two in $\kappa$ that acts on $P \psi(t)$.

Let us choose $\omega \equiv E_{0}$, so that $\omega-\lambda_{1}=-P W B P=\mathcal{O}(\kappa)$. Using (3.8), (3.10) and (3.12), we get our final version of the equation of motion for $P \psi$ :

$$
i \partial_{t} P \psi=\lambda P \psi+f
$$

where $\lambda \equiv \lambda_{1}-P W\left(\bar{H}-E_{0}-i 0\right)^{-1} P_{d} W P, f=\sum_{j=1}^{5} f_{j}$, and the $f_{j}$ 's are given by

$$
\begin{aligned}
& f_{1}(t)=P W B e^{-i \bar{H} t} \psi_{d}(0), \\
& f_{2}(t)=P W e^{-i \bar{H} t}\left(\bar{H}-E_{0}-i 0\right)^{-1} P_{d} W P \psi(0), \\
& f_{3}(t)=-i \int_{0}^{t} P W e^{-i \bar{H}(t-s)}\left(\bar{H}-E_{0}-i 0\right)^{-1} P_{d} W P W B P \psi(s) d s, \\
& f_{4}(t)=-i \int_{0}^{t} P W e^{-i \bar{H}(t-s)}\left(\bar{H}-E_{0}-i 0\right)^{-1} P_{d} W P W B \psi_{d}(s) d s, \\
& f_{5}(t)=-i \int_{0}^{t} P W B^{\prime} e^{-i \bar{H}(t-s)} P_{d} W P \psi(s) d s .
\end{aligned}
$$

The expression for $\lambda$ is analyzed in

Proposition 3.3. The expansion (2.7)-(2.8) holds for $\lambda$.

The proof is given in the appendix.

Discussion. Our proof is based on estimating Eqs. (3.4) and (3.13) (obtained estimates are then synthesized into the final theorem with the help of Eqs. (3.6)-(3.7)). Equation (3.13) is a one-iteration of Eq. (3.8). This iteration is needed so that the bounded operator $\lambda$ multiplying the vector function $P \psi$ on the r.h.s. of the equation for $P \psi$ captures the leading non-zero term of the imaginary part of the resonance. In our case, due to Assumption (A5), this term is the second order. That is why we need only one iteration. 
For the leading term of the imaginary part of a higher order (one can show that the leading term is always of an even order and positive) one should iterate Eq. (3.13) further ( $n-2$ times for order $n$ ). Controlling the resulting terms would require faster time decay of terms like $W \psi_{d}(t)$ which would result in a higher power $\alpha$ in Conditions (A1)-(A4).

3.2. Estimates of $P \psi(t)$ and $\psi_{d}(t)$ and proof of Theorem 2.1. In this section, we show the estimates given in Theorem 2.1. Due to $\psi=B\left(P \psi+\psi_{d}\right)$ (see (3.6), (3.7)) and Lemma A.1 of the appendix which proves that

$$
\left\|<A>^{-\alpha} B \phi\right\|_{\mathcal{H}} \leq C\left\|<A>^{-\alpha} \phi\right\|_{\mathcal{H}}, \quad \forall \phi \in \mathcal{H}
$$

and

$$
\left\|<A>^{-\alpha} B^{\prime} \phi\right\|_{\mathcal{H}} \leq C \kappa\left\|<A>^{-\alpha} \phi\right\|_{\mathcal{H}}, \quad \forall \phi \in \mathcal{H}
$$

it suffices to demonstrate appropriate estimates on $P \psi(t)$ and $\psi_{d}(t)$. To this end, write the integral equations for $P \psi(t)$ and $\psi_{d}(t)$ (cf. (3.13) and (3.9)):

$$
\begin{aligned}
P \psi(t) & =e^{-i \lambda t} P \psi(0)+r(t), \\
\psi_{d}(t) & =e^{-i \bar{H} t} \psi_{d}(0)+R(t),
\end{aligned}
$$

where $r(t)=\sum_{j=1}^{5} r_{j}(t)$, with

$$
r_{j}(t)=-i \int_{0}^{t} e^{-i \lambda(t-s)} f_{j}(s) d s
$$

$j=1, \ldots, 5$, and

$$
R(t)=-i \int_{0}^{t} e^{-i \bar{H}(t-s)} P_{d} W P \psi(s) d s .
$$

The strategy (see [SW3]) is the following: for $T>0$ and some $\beta \geq 0$, introduce the norms

$$
[r]_{T}=\sup _{0 \leq t \leq T}(t+1)^{\beta}\|r(t)\|_{\mathcal{H}}
$$

and

$$
[R]_{T}=\sup _{0 \leq t \leq T}(t+1)^{\beta}\left\|<A>^{-\alpha} R(t)\right\|_{\mathcal{H}}
$$

Using (3.17) and (3.18), we then show that $[r]_{T} \leq C \kappa^{1-2 \beta}$, where the constant is independent of $T, \kappa$ is sufficiently small and $0 \leq \beta<\min \{1 / 2, \alpha-2\}$. Taking $T \rightarrow \infty$ gives us the desired result: $\|r(t)\|_{\mathcal{H}} \leq C \kappa^{1-2 \beta}(t+1)^{-\beta}$. The corresponding estimate for $\left\|<A>^{-\alpha} \psi_{d}(t)\right\|_{\mathcal{H}}$ is obtained similarly. These estimates and Eqs. (3.6), (3.7) and (3.15) imply the proof of Theorem 2.1 .

\section{Ingredients of the estimations.}

- The basic tool in the estimations is the local decay Assumption (A4), and its integrated version given in Proposition 3.2(iii). 
- In order to estimate $\left\|e^{-i \lambda t}\right\|$, we use the expansion for $\lambda$ given in Proposition 3.3. We have

$$
\left\|e^{-i \lambda t}\right\| \leq e^{C \kappa^{3} t} e^{-C_{0} \kappa^{2} t}
$$

In order to prove this inequality, we use the differential inequality

$$
\begin{aligned}
\frac{d}{d t}\left\|e^{-i \lambda t} u\right\|^{2} & =\left\langle e^{-i \lambda t} u, i\left(\lambda^{*}-\lambda\right) e^{-i \lambda t} u\right\rangle \\
& \leq 2 \sup (\operatorname{Im} \lambda)\left\|e^{-i \lambda t} u\right\|^{2},
\end{aligned}
$$

and the initial condition $\left.\left\|e^{-i \lambda t} u\right\|\right|_{t=0}=\|u\|$ to obtain that $\left\|e^{-i \lambda t}\right\| \leq\left\|e^{\operatorname{Im} \lambda t}\right\|$. (The latter inequality can also be derived by taking the norm of the Trotter representation of $e^{-i \lambda t}=\lim _{n \rightarrow \infty}\left[e^{-i \operatorname{Re} \lambda t / n} e^{\operatorname{Im} \lambda t / n}\right]^{n}$.) Using that $\operatorname{Im} \lambda=-\Gamma+\mathcal{O}\left(\kappa^{3}\right)$ and that the spectrum of $\Gamma$ is bounded below by $C_{0} \kappa^{2}$, we arrive at (3.21). Setting $\gamma \equiv C_{0} \kappa^{2} / 2$, we derive from (3.21) for $\kappa$ small:

$$
\left\|e^{-i \lambda t}\right\| \leq e^{-\gamma t}, \quad t \geq 0 .
$$

- We have the following uniform bounds in $t \geq 0$. a) For $0 \leq \beta \leq \sigma$ :

$$
(t+1)^{\beta} \int_{0}^{t} e^{-\gamma(t-s)}(s+1)^{-\sigma} d s \leq C\left(1+\gamma^{-1-\beta}\right) \leq C \kappa^{-2-2 \beta}
$$

If $0 \leq \beta \leq \sigma-1$ and $\sigma>1$, then the r.h.s. above can be replaced by $C\left(1+\gamma^{-\beta}\right) \leq$ $C \kappa^{-\overline{2} \beta}$.

b) For $\sigma>1,0 \leq \beta \leq \sigma-1$ :

$$
(t+1)^{\beta} \int_{0}^{t}(t-s+1)^{-\sigma}(s+1)^{-\beta} d s \leq C .
$$

Estimations. We use the above mentioned points and the condition $\alpha-2 \geq \beta$ to estimate $\left\|r_{j}(t)\right\|_{\mathcal{H}}$, for $j=1, \ldots, 5$.

$$
\text { 1) } \begin{aligned}
\left\|r_{1}(t)\right\|_{\mathcal{H}} & \leq \int_{0}^{t} e^{-\gamma(t-s)}\left\|P W B e^{-i \bar{H} s} \psi_{d}(0)\right\|_{\mathcal{H}} d s \\
& \leq C\left\|<A>^{\alpha} \bar{P} \psi_{0}\right\|_{\mathcal{H}} \kappa \int_{0}^{t} e^{-\gamma(t-s)}(s+1)^{-\alpha} d s \\
& \leq C\left\|<A>^{\alpha} \bar{P} \psi_{0}\right\|_{\mathcal{H}} \kappa^{1-2 \beta}(1+t)^{-\beta}, t \geq 0 .
\end{aligned}
$$

In the second step, we used (3.15) and the local decay estimate (A4).

$$
\text { 2) } \begin{aligned}
\left\|r_{2}(t)\right\|_{\mathcal{H}} & \leq C\left\|P \psi_{0}\right\|_{\mathcal{H}} \kappa^{2} \int_{0}^{t} e^{-\gamma(t-s)}(s+1)^{-\alpha+1} d s \\
& \leq C\left\|P \psi_{0}\right\|_{\mathcal{H}} \kappa^{2-2 \beta}(t+1)^{-\beta}, t \geq 0 .
\end{aligned}
$$

3) $\left\|r_{3}(t)\right\|_{\mathcal{H}} \leq C \kappa^{3} \int_{0}^{t} e^{-\gamma(t-s)} \int_{0}^{s}(s-\tau+1)^{-\alpha+1}\|P \psi(\tau)\|_{\mathcal{H}} d \tau d s$. 
We split $\|P \psi(\tau)\|_{\mathcal{H}}$ as

$$
\begin{aligned}
\|P \psi(\tau)\|_{\mathcal{H}} & \leq e^{-\gamma \tau}\left\|P \psi_{0}\right\|_{\mathcal{H}}+\|r(\tau)\|_{\mathcal{H}} \leq e^{-\gamma \tau}\left\|P \psi_{0}\right\|_{\mathcal{H}}+[r]_{T}(\tau+1)^{-\beta} \\
& \leq C\left(\gamma^{-\beta}\left\|P \psi_{0}\right\|_{\mathcal{H}}+[r]_{T}\right)(\tau+1)^{-\beta}
\end{aligned}
$$

where we used $(\gamma \tau)^{\beta} e^{-\gamma \tau} \leq C$, uniformly in $\gamma, \tau \geq 0$. So

$$
\begin{aligned}
\left\|r_{3}(t)\right\|_{\mathcal{H}} & \leq C \kappa^{3}\left(\kappa^{-2 \beta}\left\|P \psi_{0}\right\|_{\mathcal{H}}+[r]_{T}\right) \int_{0}^{t} e^{-\gamma(t-s)}(s+1)^{-\beta} d s \\
& \leq C \kappa^{1-2 \beta}\left(\kappa^{-2 \beta}\left\|P \psi_{0}\right\|_{\mathcal{H}}+[r]_{T}\right)(t+1)^{-\beta}
\end{aligned}
$$

We decompose the last term in the integral as

$$
\begin{aligned}
\left\|<A>^{-\alpha} \psi_{d}(\tau)\right\|_{\mathcal{H}} & \leq\left\|<A>^{-\alpha} e^{-i \bar{H} \tau} \psi_{d}(0)\right\|_{\mathcal{H}}+\left\|<A>^{-\alpha} R(\tau)\right\|_{\mathcal{H}} \\
& \leq C(\tau+1)^{-\alpha}\left\|<A>^{\alpha} \bar{P} \psi_{0}\right\|_{\mathcal{H}}+(\tau+1)^{-\beta}[R]_{T}
\end{aligned}
$$

Using this decomposition in the double-integral, we get

$$
\left\|r_{4}(t)\right\|_{\mathcal{H}} \leq C\left(\left\|<A>^{\alpha} \bar{P} \psi_{0}\right\|_{\mathcal{H}} \kappa^{3-2 \beta}+[R]_{T} \kappa^{1-2 \beta}\right)(t+1)^{-\beta} .
$$

$$
\text { 5) } \begin{aligned}
\left\|r_{5}(t)\right\|_{\mathcal{H}} & \leq C \kappa^{3} \int_{0}^{t} e^{-\gamma(t-s)} \int_{0}^{s}(s-\tau+1)^{-\alpha}\|P \psi(\tau)\|_{\mathcal{H}} d \tau d s \\
& \leq C \kappa^{1-2 \beta}\left(\kappa^{-2 \beta}\left\|P \psi_{0}\right\|_{\mathcal{H}}+[r]_{T}\right)(t+1)^{-\beta} .
\end{aligned}
$$

Summing (3.23), (3.24), (3.26), (3.27) and (3.28), we find for $0 \leq t \leq T$ :

$$
\begin{aligned}
(t+1)^{\beta}\|r(t)\|_{\mathcal{H}} \leq & C[r]_{T} \kappa^{1-2 \beta}+C[R]_{T} \kappa^{1-2 \beta} \\
& +C\left\|P \psi_{0}\right\|_{\mathcal{H}} \kappa^{1-4 \beta}+C\left\|<A>^{\alpha} \bar{P} \psi_{0}\right\|_{\mathcal{H}} \kappa^{1-2 \beta}
\end{aligned}
$$

where the r.h.s. is independent of $t$. In order to close the estimates, we express $[R]_{T}$ in terms of $[r]_{T}$. From (3.18), we get

$$
\begin{aligned}
\left\|<A>^{-\alpha} R(t)\right\|_{\mathcal{H}} & \leq C \kappa \int_{0}^{t}(t-s+1)^{-\alpha}\|P \psi(s)\|_{\mathcal{H}} d s \\
& \leq C\left(\kappa^{1-2 \beta}\left\|P \psi_{0}\right\|_{\mathcal{H}}+[r]_{T} \kappa\right)(t+1)^{-\beta}
\end{aligned}
$$

and thus from (3.20): $[R]_{T} \leq C \kappa^{1-2 \beta}\left\|P \psi_{0}\right\|_{\mathcal{H}}+C \kappa[r]_{T}$. Taking the supremum over $t \in[0, T]$ in (3.29), and replacing $[R]_{T}$ in (3.29) by the last estimate, we get

$$
[r]_{T} \leq C[r]_{T} \kappa^{1-2 \beta}+C\left\|P \psi_{0}\right\|_{\mathcal{H}} \kappa^{1-4 \beta}+C\left\|<A>^{\alpha} \bar{P} \psi_{0}\right\|_{\mathcal{H}} \kappa^{1-2 \beta} .
$$

We can now isolate $[r]_{T}$ in this inequality if $\beta<1 / 2$, and for $\kappa$ small enough. We get

$$
[r]_{T} \leq C\left\|P \psi_{0}\right\|_{\mathcal{H}} \kappa^{1-4 \beta}+C\left\|<A>^{\alpha} \bar{P} \psi_{0}\right\|_{\mathcal{H}} \kappa^{1-2 \beta} .
$$


The estimate is uniform in $T$, and taking $T \rightarrow \infty$ yields

$$
\|r(t)\|_{\mathcal{H}} \leq C \kappa^{1-4 \beta}(t+1)^{-\beta} .
$$

Similarly, using $[r]_{T} \leq C \kappa^{1-4 \beta}$ in (3.30) gives

$$
\left\|<A>^{-\alpha} R(t)\right\|_{\mathcal{H}} \leq C \kappa^{1-2 \beta}(t+1)^{-\beta} .
$$

The estimates given in Remark 2 after Theorem 2.1 are immediate from (3.30) and (3.32).

\section{The Mourre Estimate for Reduced Operators and Local Decay}

4.1. The Mourre estimate. In this section, we derive the strict Mourre estimate for the operator $\bar{H} \equiv \bar{P} H \bar{P}$ from the Mourre estimate for $H_{0}$ and use the former to prove the local decay for the operator $\bar{H}$. We consider perturbations $W$ of the form $W=\kappa^{\prime} U$, where $U$ is fixed, and $\kappa^{\prime}$ is assumed to be sufficiently small. The main result of this section is related to some results in [AHS]. It is given in the following

Theorem 4.1. Suppose that $A P$ and $\left[H_{0}, A\right]\left(H_{0}+i\right)^{-1}$ are bounded, and that $A W P$, $W\left(H_{0}+i\right)^{-1}$ and $[W, A]\left(H_{0}+i\right)^{-1}$ are $\mathcal{O}\left(\kappa^{\prime}\right)$. Moreover, suppose that there is a neighbourhood $\Delta_{1}$ of $E_{0} \neq 0$ such that

$$
E_{\Delta_{1}}\left(H_{0}\right) i\left[H_{0}, A\right] E_{\Delta_{1}}\left(H_{0}\right) \geq \theta_{1} E_{\Delta_{1}}\left(H_{0}\right)+K,
$$

where $\theta_{1}>0$ and $K$ is a compact operator. Then $\forall \epsilon>0 \exists C(\epsilon)>0$ such that

$$
E_{\Delta_{3}}(\bar{H}) i[\bar{H}, A] E_{\Delta_{3}}(\bar{H}) \geq\left(\theta_{1}-\epsilon\right) E_{\Delta_{3}}(\bar{H}),
$$

for any neighbourhood $\Delta_{3}$ of $E_{0}, \Delta_{3} \subset \Delta_{1}$, provided $\left|\Delta_{3}\right| \leq C(\epsilon)$, and $\kappa^{\prime} \leq C(\epsilon)$. In particular, $\left|\Delta_{3}\right|$ is so small that $0 \notin \Delta_{3}$.

Proof. We divide the proof into two steps. In Step 1, we pass from the Mourre estimate for $H_{0}$ to a strong Mourre estimate for $\bar{H}_{0}$, in an appropriate interval $\Delta_{2}, 0 \notin \Delta_{2} \subset \Delta_{1}$. This is done by shrinking $\Delta_{2}$ around $E_{0}$ as to make the contribution of the compact operator $K$ arbitrarily small. In the second step, we pass from the strong Mourre estimate for $\bar{H}_{0}$ to one for $\bar{H}$.

Step 1. We show that $\forall \epsilon_{1}>0 \exists C_{1}\left(\epsilon_{1}\right)>0$ such that

$$
E_{\Delta_{2}}\left(\bar{H}_{0}\right) i\left[\bar{H}_{0}, A\right] E_{\Delta_{2}}\left(\bar{H}_{0}\right) \geq\left(\theta_{1}-\epsilon_{1}\right) E_{\Delta_{2}}\left(\bar{H}_{0}\right)
$$

for any neighbourhood $\Delta_{2}$ of $E_{0}$ such that $\Delta_{2} \subset \Delta_{1}$, and $\left|\Delta_{2}\right| \leq C_{1}\left(\epsilon_{1}\right)$.

Let $\Delta_{2}$ be an open set containing $E_{0}$ but not 0 , and $\Delta_{2} \subset \Delta_{1}$. Applying $\bar{P} E_{\Delta_{2}}\left(\bar{H}_{0}\right)=$ $E_{\Delta_{2}}\left(\bar{H}_{0}\right)$ to both sides of Eq. (4.1) and using $E_{\Delta_{1}}\left(H_{0}\right) \bar{P}=E_{\Delta_{1}}\left(\bar{H}_{0}\right) \bar{P}=E_{\Delta_{1}}\left(\bar{H}_{0}\right)$, we obtain

$$
E_{\Delta_{2}}\left(\bar{H}_{0}\right) i\left[\bar{H}_{0}, A\right] E_{\Delta_{2}}\left(\bar{H}_{0}\right) \geq \theta_{1} E_{\Delta_{2}}\left(\bar{H}_{0}\right)+E_{\Delta_{2}}\left(\bar{H}_{0}\right) K E_{\Delta_{2}}\left(\bar{H}_{0}\right),
$$

where the last term on the r.h.s. can be made arbitrarily small by shrinking $\Delta_{2}$ around $E_{0}\left(\bar{H}_{0}\right.$ has no eigenvalues in a neighbourhood of $E_{0}$ if $E_{0} \neq 0$, thus $E_{\Delta_{2}}\left(\bar{H}_{0}\right) \rightarrow 0$ strongly; and $K$ is compact). This shows (4.3). 
Step 2. Let now $\Delta_{2}$ satisfy (4.3). We show that $\forall \epsilon_{2}>0 \exists C_{2}\left(\epsilon_{2}\right)>0$ such that

$$
E_{\Delta_{3}}(\bar{H}) i[\bar{H}, A] E_{\Delta_{3}}(\bar{H}) \geq\left(\theta_{1}-\epsilon_{1}-\epsilon_{2}\right) E_{\Delta_{3}}(\bar{H}),
$$

for any neighbourhood $\Delta_{3}$ of $E_{0}$ such that $\bar{\Delta}_{3} \subset \Delta_{2}$, provided $\kappa^{\prime} \leq C_{2}\left(\epsilon_{2}\right)$.

We have

$$
[\bar{H}, A]=\left[\bar{H}_{0}, A\right]+[\bar{W}, A]
$$

We claim

$$
E_{\Delta_{2}}(\bar{H})[\bar{W}, A] E_{\Delta_{2}}(\bar{H})=\mathcal{O}\left(\kappa^{\prime}\right) .
$$

To prove the last estimate, write

$$
[\bar{W}, A]=[W, A]+[P W P, A]-[P W, A]-[W P, A] .
$$

The last three commutators are $\mathcal{O}\left(\kappa^{\prime}\right)$ since $\|A P\|<\infty$ and $\|A W P\|=O\left(\kappa^{\prime}\right)$. The fact that $[W, A](\bar{H}+i)^{-1}=\mathcal{O}\left(\kappa^{\prime}\right)$ follows from the assumption $[W, A]\left(H_{0}+i\right)^{-1}=\mathcal{O}\left(\kappa^{\prime}\right)$ and $W\left(H_{0}+i\right)^{-1}=\mathcal{O}\left(\kappa^{\prime}\right)$.

Let us now examine $E_{\Delta_{3}}(\bar{H}) i\left[\bar{H}_{0}, A\right] E_{\Delta_{3}}(\bar{H})$, where $\Delta_{3}$ is a neighbourhood of $E_{0}$, and $\bar{\Delta}_{3} \subset \Delta_{2}$. Let $h \in C_{0}^{\infty}\left(\Delta_{2}\right)$ be such that $h=1$ on $\Delta_{3}$. We have $h(\bar{H})=h\left(\bar{H}_{0}\right)+\mathcal{I}$, where

$$
\mathcal{I} \equiv-\int\left(\bar{H}_{0}-z\right)^{-1} \bar{W}(\bar{H}-z)^{-1} d \tilde{h}(z)
$$

and since $\left[\bar{H}_{0}, A\right]\left(\bar{H}_{0}+i\right)^{-1}$ is bounded, and both $\mathcal{I}$ and $\left(\bar{H}_{0}+i\right) \mathcal{I}$ are $\mathcal{O}\left(\kappa^{\prime}\right)$, we get

$$
\begin{aligned}
& E_{\Delta_{3}}(\bar{H}) i\left[\bar{H}_{0}, A\right] E_{\Delta_{3}}(\bar{H}) \\
& =E_{\Delta_{3}}(\bar{H}) h(\bar{H}) i\left[\bar{H}_{0}, A\right] h(\bar{H}) E_{\Delta_{3}}(\bar{H}) \\
& =E_{\Delta_{3}}(\bar{H}) h\left(\bar{H}_{0}\right) i\left[\bar{H}_{0}, A\right] h\left(\bar{H}_{0}\right) E_{\Delta_{3}}(\bar{H})+\mathcal{O}\left(\kappa^{\prime}\right) .
\end{aligned}
$$

Using (4.3) and $h^{2}\left(\bar{H}_{0}\right)=h^{2}(\bar{H})+\mathcal{O}\left(\kappa^{\prime}\right)$, we estimate the first term on the r.h.s. of (4.8) from below by

$$
\left(\theta_{1}-\epsilon_{1}\right) E_{\Delta_{3}}(\bar{H}) h^{2}\left(\bar{H}_{0}\right) E_{\Delta_{3}}(\bar{H})=\left(\theta_{1}-\epsilon_{1}\right) E_{\Delta_{3}}(\bar{H})+\mathcal{O}\left(\kappa^{\prime}\right)
$$

This together with (4.8) implies

$$
E_{\Delta_{3}}(\bar{H}) i\left[\bar{H}_{0}, A\right] E_{\Delta_{3}}(\bar{H}) \geq\left(\theta_{1}-\epsilon_{1}\right) E_{\Delta_{3}}(\bar{H})+\mathcal{O}\left(\kappa^{\prime}\right) .
$$

Multiplying (4.6) from both sides by $E_{\Delta_{3}}(\bar{H})$ and taking into account (4.7) and (4.9) yields the desired result (4.5).

\subsection{Local decay.}

Theorem 4.2. Suppose (A3) and (4.2) hold, $\left\|W\left(H_{0}+i\right)^{-1}\right\|<1$, and $\left(H_{0}+i\right)^{m} a d_{A}^{(r)}(P)$ $\left(H_{0}+i\right)^{-m}$ are bounded for $m=0,1$ and $r \leq n$. Then there is an interval $\Delta^{\prime}, E_{0} \in$ $\Delta^{\prime} \subset \Delta_{3}$ such that the local decay estimate (A4) holds. 
Proof. We verify first that we have the upper bound (uniformly in $W$ ):

$$
\left\|a d_{A}^{(k)}(\bar{H})(\bar{H}+i)^{-1}\right\| \leq C, \quad k=1, \ldots, n .
$$

Expanding the multicommutator of order $k$ gives

$$
a d_{A}^{(k)}(\bar{H})=a d_{A}^{(k)}(\bar{P} H \bar{P})=\sum_{r_{1}+r_{2}+r_{3}=k} C_{r_{1}, r_{2}, r_{3}} a d_{A}^{\left(r_{1}\right)}(\bar{P}) a d_{A}^{\left(r_{2}\right)}(H) a d_{A}^{\left(r_{3}\right)}(\bar{P}) .
$$

Equation (4.10) follows since all the operators

$$
a d_{A}^{\left(r_{1}\right)}(\bar{P}), a d_{A}^{\left(r_{2}\right)}(H)\left(H_{0}+i\right)^{-1},\left(H_{0}+i\right) a d_{A}^{\left(r_{3}\right)}(\bar{P})\left(H_{0}+i\right)^{-1},
$$

and $\left(H_{0}+i\right)(\bar{H}+i)^{-1}$ are bounded (the last fact follows from $\left.\left\|W\left(H_{0}+i\right)^{-1}\right\|<1\right)$. Theorem 4.1 together with (4.10) imply, due to a result of [HSS] that

$$
\left\|<A>^{-\alpha} e^{-i \bar{H} t} P_{d} \phi\right\|_{\mathcal{H}} \leq \text { const. }(t+1)^{-\alpha}\left\|<A>^{\alpha} \bar{P} \phi\right\|_{\mathcal{H}}, t \geq 0 .
$$

The constant depends on $\bar{H}$ (and $\kappa$ ) only through $\left\|a d_{A}^{(k)}(\bar{H})(\bar{H}+i)^{-1}\right\|, k=1, \ldots, n$.

\section{Proof of Theorem 2.3}

- We show (A1), i.e. $\left\|<A>^{\alpha} P\right\|<\infty$. From $\left\|<A>^{\alpha} P\right\| \leq C\left(1+\left\|A^{\alpha} P\right\|\right)$, we see that it is enough to show that $A^{n} P$ is bounded, for some integer $n \geq \alpha$. Now

$$
A^{n}=\left(\frac{1}{2}(x \cdot p+p \cdot x)\right)^{n}=\sum_{k=0}^{n} c_{k}(x \cdot p)^{k} .
$$

But $(x \cdot p)^{k} P$ is bounded by (2.12), $k \leq n$ : in fact, $(x \cdot p)^{k}$ is a sum of terms of the form $x^{\mathbf{m}} p^{\mathbf{m}}$, where $\mathbf{m} \equiv\left(m_{1}, \ldots, m_{\nu}\right)$ are multi-indices with $|\mathbf{m}| \equiv \sum_{j=1}^{\nu} m_{i} \leq k$, $x^{\mathbf{m}}=x_{1}^{m_{1}} \cdots x_{\nu}^{m_{\nu}}$, and $p^{\mathbf{m}}=(-i)^{|\mathbf{m}|} \partial_{x_{1}}^{m_{1}} \cdots \partial_{x_{\nu}}^{m_{\nu}}$, with $\nu=\operatorname{dim} X$.

- (A2) follows directly from $W=\kappa^{\prime} U$ and (2.11).

- We show that (A3) is satisfied. From

$$
a d_{A}^{(k)}(H)=(-2 i)^{k} p^{2}+i^{k}(x \cdot \nabla)^{k} V+i^{k}(x \cdot \nabla)^{k} W,
$$

$W=\kappa^{\prime} U$ and (S2), it is clear that $a d_{A}^{(k)}(H)$ is $H$-bounded, uniformly in $\kappa^{\prime}$.

- We show now the local decay estimate (A4). Equation (2.11) shows that $A W P=$ $\mathcal{O}\left(\kappa^{\prime}\right)$, and (S2)(ii) with $k=0,1$ gives that $W\left(H_{0}+i\right)^{-1}$ and $[W, A]\left(H_{0}+i\right)^{-1}$ are $\mathcal{O}\left(\kappa^{\prime}\right)$. Boundedness of $A P$ follows from $\left\|<A>^{\alpha} P\right\|<\infty$, which we have shown above, and $\left[H_{0}, A\right]\left(H_{0}+i\right)^{-1}$ is bounded by (S2)(i) with $k=1$. This together with (S3) shows that the conditions of Theorem 4.1 are met. Due to Theorem 4.2, it is then enough to check that $\left(H_{0}+i\right)^{m} a d_{A}^{(r)}(P)\left(H_{0}+i\right)^{-m}$ is bounded for $r \leq n, m=0,1$. We do this now.

Let $m=0$. Clearly $a d_{A}^{(0)}(P)=P$ is bounded. If $r \geq 1$, then $a d_{A}^{(r)}(P)=a d_{(x \cdot p)}^{(r)}(P)$. This multi-commutator is a sum of terms of the form $(x \cdot p)^{l} P(x \cdot p)^{m}, l+m=r$. It is thus enough to show that $x^{\mathbf{l}} p^{\mathbf{l}} P p^{\mathbf{m}} x^{\mathbf{m}}$ is bounded, $|\mathbf{I}|+|\mathbf{m}| \leq r, r \leq n$. This is 
guaranteed by (2.12). We conclude that $a d_{A}^{(r)}(P)$ is bounded (and does not depend on $\left.\kappa^{\prime}\right)$.

Let $m=1$. We write

$$
\left(H_{0}+i\right) a d_{A}^{(r)}(P)\left(H_{0}+i\right)^{-1}=a d_{A}^{(r)}(P)+\left[H_{0}, a d_{A}^{(r)}(P)\right]\left(H_{0}+i\right)^{-1} .
$$

For $r=0$, the commutator is zero. For $r>0$, the general term in the commutator is of the form

$$
H_{0}(x \cdot p)^{l} P(x \cdot p)^{m}-(x \cdot p)^{l} P(x \cdot p)^{m} H_{0} .
$$

Because $H_{0}$ is $p^{2}$-bounded, it is enough to show that $p^{2}(x \cdot p)^{l} P(x \cdot p)^{m}$ is bounded for any $l, m, l+m \leq n$. This is again ensured by (2.12).

- (A5) follows immediately from (S4).

\section{Proof of Theorem 2.5}

The proof of Theorem 2.5 is analogous to the proof of Theorem 2.1 for a non-degenerate eigenvalue $E_{0}$. In the quasiclassical case however, we must keep track of the parameter $\hbar$. The delicate point in the proof of Theorem 2.5 is to show local decay. The latter is deduced from the quasiclassical Mourre estimate (cf. [G, Gr, HN]). This estimate is of an independent interest. We begin with it.

6.1. The quasiclassical Mourre estimate.

Theorem 6.1. Assume the non-trapping Condition (T3). Then $\forall \epsilon>0$, there is a $C_{\epsilon}>0$ and a neighbourhood $\Delta_{1}$ of $E_{0} / \hbar$ (with $\left|\Delta_{1}\right|$ independent of $\hbar$ ) such that for $\hbar \leq C_{\epsilon}$, we have

$$
E_{\hbar \Delta_{1}}(\bar{H}) \frac{i}{\hbar}[\bar{H}, A] E_{\hbar \Delta_{1}}(\bar{H}) \geq(\theta-\epsilon) E_{\hbar \Delta_{1}}(\bar{H}) .
$$

Proof. We write $[\bar{H}, A]=\bar{P}[H, A] \bar{P}+\bar{P} H[P, A]+[P, A] H \bar{P}$. Since $P A$ and $A P$ are bounded, and $\bar{P} H P=\bar{P} W P=\mathcal{O}(\kappa)$, we get $\bar{P}[\bar{H}, A] \bar{P}=\bar{P}[H, A] \bar{P}+\mathcal{O}(\kappa)$. Let $\Delta_{1}$ be an interval containing $E_{0} / \hbar$, and of fixed length as specified in Proposition 6.2(i) below, and such that $0 \notin \hbar \Delta_{1}$. Then we have $E_{\hbar \Delta_{1}}(\bar{H})=E_{\hbar \Delta_{1}}(\bar{H}) \bar{P}$, and hence

$$
E_{\hbar \Delta_{1}}(\bar{H}) \frac{i}{\hbar}[\bar{H}, A] E_{\hbar \Delta_{1}}(\bar{H})=E_{\hbar \operatorname{delta} a_{1}}(\bar{H}) \frac{i}{\hbar}[H, A] E_{\hbar \Delta_{1}}(\bar{H})+\mathcal{O}(\kappa) .
$$

Now $\frac{i}{\hbar}[H, A]=2\left(H-E_{0}\right)+2\left(E_{0}-V(x)\right)-x \cdot \nabla V(x)$, so with $E_{0} \geq V(0)$, it follows that

$$
\begin{aligned}
& E_{\hbar \Delta_{1}}(\bar{H}) \frac{i}{\hbar}[\bar{H}, A] E_{\hbar \Delta_{1}}(\bar{H}) \\
& \geq E_{\hbar \Delta_{1}}(\bar{H})(2(V(0)-V(x))-x \cdot \nabla V(x)) E_{\hbar \Delta_{1}}(\bar{H})+\mathcal{O}(\hbar) .
\end{aligned}
$$

Let $\mathcal{N}$ be a bounded neighbourhood of $0 \in \mathbb{R}^{d}$ on which $V(x)=V_{0}(x)$ (see (ii) in Paragraph 2.3.2). Let $\partial \mathcal{N} \equiv \overline{\mathcal{N}} \backslash \mathcal{N}^{\circ}$ be the boundary of $\mathcal{N}$. We put $\delta_{1}=\min _{x \in \partial \mathcal{N}}(V(x)-$ $V(0)$ ), and $\delta_{2}=\min \left\{\delta_{1}, \delta\right\} / 2$, where $\delta$ is given in (T3). The sets $\Omega_{\delta}^{e x t}, \mathcal{N}$ and $\Omega_{\delta_{2}}^{C} \equiv$ $\left\{x \in \mathbb{R}^{d} \mid V(x) \geq \delta_{2}\right\}$ cover $\mathbb{R}^{d}$. 
We introduce a $C^{\infty}$-decomposition of unity: $1=\chi_{1}(x)+\chi_{2}(x)+\chi_{3}(x)$, such that supp $\chi_{1} \subset \mathcal{N}$, supp $\chi_{2} \subset \Omega_{\delta}^{\text {ext }}$, supp $\chi_{3} \subset \Omega_{\delta_{2}}^{C}$. We estimate the r.h.s. of (6.3) on the supports of $\chi_{1}, \chi_{2}$ and $\chi_{3}$. We have

$$
\chi_{3}(x) E_{\hbar \Delta_{1}}(H)=\mathcal{O}\left(e^{-\rho / \hbar}\right),
$$

for some $\rho>0$ (see [Gr], Lemma 6). On the support of $\chi_{1}$ we have $V(x)=V_{0}(x)$, suggesting that $\chi_{1} E_{\hbar \Delta_{1}}(\bar{H})$ is close to $\chi_{1} E_{\hbar \Delta_{1}}\left(\bar{H}_{0}\right)$, which in turn is zero if $\hbar \Delta_{1}$ does not contain any eigenvalues of $\bar{H}_{0}$. We have in fact

Proposition 6.2. There is a neighbourhood $\hbar \Delta_{1}$ of $E_{0}$, such that $\left|\Delta_{1}\right|$ is independent of $\hbar\left(\left|\Delta_{1}\right|\right.$ depends only on the second derivatives of $V$ at the origin), and such that

(i) $\hbar \Delta_{1} \cap \sigma\left(H_{0}\right)=\left\{E_{0}\right\}$,

(ii) $\chi_{1} E_{\hbar \Delta_{1}}(\bar{H})=\mathcal{O}\left(\hbar^{1 / 2}\right)$.

The proof is given below. Putting $1=\chi_{1}(x)+\chi_{2}(x)+\chi_{3}(x)$ in front of the last factor $E_{\hbar \Delta_{1}}(\bar{H})$ in the r.h.s. of (6.3), we then get, using (6.4), Proposition 6.2 and the non-trapping Condition (T3):

$$
\begin{aligned}
& E_{\hbar \Delta_{1}}(\bar{H}) \frac{i}{\hbar}[\bar{H}, A] E_{\hbar \Delta_{1}}(\bar{H}) \\
& \geq E_{\hbar \Delta_{1}}(\bar{H})(2(V(0)-V(x))-x \cdot \nabla V(x))\left(\chi_{1}(x)+\chi_{2}(x)\right) E_{\hbar \Delta_{1}}(\bar{H})+\mathcal{O}(\hbar) \\
& \geq E_{\hbar \Delta_{1}}(\bar{H})\left(\theta \chi_{2}(x)+\mathcal{O}\left(\hbar^{1 / 2}\right)\right) E_{\hbar \Delta_{1}}(\bar{H})+\mathcal{O}(\hbar) \\
& \geq E_{\hbar \Delta_{1}}(\bar{H})\left(\theta+\mathcal{O}\left(\hbar^{1 / 2}\right)\right) E_{\hbar \Delta_{1}}(\bar{H}) .
\end{aligned}
$$

Proof of Proposition 6.2.. (i) is a simple consequence of the harmonic approximation. by

In order to prove (ii), we introduce the unitary transformation $\mathcal{U}$ on $L^{2}\left(\mathbb{R}^{d}\right)$ defined

$$
(\mathcal{U} \psi)(x)=\hbar^{d / 4} \psi\left(\hbar^{1 / 2} x\right), \quad \psi \in L^{2}\left(\mathbb{R}^{d}\right) .
$$

It is easily seen that $\mathcal{U} H \mathcal{U}^{-1}=\hbar H^{\prime}, \mathcal{U} H_{0} \mathcal{U}^{-1}=\hbar H_{0}^{\prime}$, where the rescaled Hamiltonians are given by $H^{\prime}=-\Delta+\hbar^{-1} V\left(\hbar^{1 / 2} x\right)$, and $H_{0}^{\prime}=-\Delta+\hbar^{-1} V_{0}\left(\hbar^{1 / 2} x\right)$. The spectra are related as $\sigma(H)=\hbar \sigma\left(H^{\prime}\right)$, and $\sigma\left(H_{0}\right)=\hbar \sigma\left(H_{0}^{\prime}\right)$. For a function $g$ of $H$, we have $\mathcal{U} g(H) \mathcal{U}^{-1}=g\left(\hbar H^{\prime}\right)$. We let $\overline{H^{\prime}} \equiv \overline{P^{\prime}} H^{\prime} \overline{P^{\prime}}$, where $\overline{P^{\prime}}=1-P^{\prime}, P^{\prime}=\mathcal{U} P \mathcal{U}^{-1}$, and $\overline{H_{0}^{\prime}} \equiv \overline{P^{\prime}} H_{0}^{\prime} \overline{P^{\prime}}$.

Pick now $g \in C_{0}^{\infty}$ such that $g=1$ on $\Delta_{1}$, and suppg $\cap \sigma\left(H_{0}^{\prime}\right)=\left\{E_{0} / \hbar\right\}$ (this is possible by (i)). Since $g\left(\overline{H_{0}^{\prime}}\right)=0$, we have

$$
\begin{aligned}
& \chi_{1}\left(\hbar^{1 / 2} x\right) g\left(\overline{H^{\prime}}\right)=\chi_{1}\left(\hbar^{1 / 2} x\right)\left(g\left(\overline{H^{\prime}}\right)-g\left(\overline{H_{0}^{\prime}}\right)\right) \\
& =-\chi_{1}\left(\hbar^{1 / 2} x\right) \int\left(\overline{H_{0}^{\prime}}-z\right)^{-1} \overline{P^{\prime}} \frac{1}{\hbar} W\left(\hbar^{1 / 2} x\right) \overline{P^{\prime}}\left(\overline{H^{\prime}}-z\right)^{-1} d \tilde{g} \\
& =-\chi_{1}\left(\hbar^{1 / 2} x\right) \int\left(H_{0}^{\prime}-z\right)^{-1} \frac{1}{\hbar} W\left(\hbar^{1 / 2} x\right) \overline{P^{\prime}}\left(\overline{H^{\prime}}-z\right)^{-1} d \tilde{g}+\mathcal{O}\left(\hbar^{-1} \kappa\right) .
\end{aligned}
$$

In the last step, we used

$$
P^{\prime} \frac{1}{\hbar} W\left(\hbar^{1 / 2} x\right)=\frac{1}{\hbar} \mathcal{U} P W \mathcal{U}^{-1}=\mathcal{O}\left(\hbar^{-1} \kappa\right) .
$$


The r.h.s. of (6.7) is now shown to be small by commuting $\chi_{1}\left(\hbar^{1 / 2} x\right)$ through the resolvent $\left(H_{0}^{\prime}-z\right)^{-1}$ to the right, and using $\chi_{1}\left(\hbar^{1 / 2} x\right) W\left(\hbar^{1 / 2} x\right)=0$ :

$$
\begin{aligned}
\chi_{1}\left(\hbar^{1 / 2} x\right)\left(H_{0}^{\prime}-z\right)^{-1} \frac{1}{\hbar} W\left(\hbar^{1 / 2} x\right) \\
=\left(H_{0}^{\prime}-z\right)^{-1}\left[-\Delta, \chi_{1}\left(\hbar^{1 / 2} x\right)\right]\left(H_{0}^{\prime}-z\right)^{-1} \frac{1}{\hbar} W\left(\hbar^{1 / 2} x\right) \\
=-2 \hbar^{1 / 2}\left(H_{0}^{\prime}-z\right)^{-1} \nabla \cdot\left(\nabla \chi_{1}\right)\left(\hbar^{1 / 2} x\right)\left(H_{0}^{\prime}-z\right)^{-1} \frac{1}{\hbar} W\left(\hbar^{1 / 2} x\right) \\
+\hbar\left(H_{0}^{\prime}-z\right)^{-1}\left(\Delta \chi_{1}\right)\left(\hbar^{1 / 2} x\right)\left(H_{0}^{\prime}-z\right)^{-1} \frac{1}{\hbar} W\left(\hbar^{1 / 2} x\right) .
\end{aligned}
$$

Notice that $\left\|\left(H_{0}^{\prime}-z\right)^{-1} \nabla\right\| \leq C|\operatorname{Im} z|^{-1}$, uniformly in $\hbar$, and that

$$
\begin{aligned}
\left(H_{0}^{\prime}-z\right)^{-1} \frac{1}{\hbar} W\left(\hbar^{1 / 2} x\right) \overline{P^{\prime}}\left(\overline{H^{\prime}}-z\right)^{-1} & =\left(\overline{H_{0}^{\prime}}-z\right)^{-1}-\left(\overline{H^{\prime}}-z\right)^{-1}+\mathcal{O}\left(\hbar^{-1} \kappa\right) \\
& =\mathcal{O}\left(|\operatorname{Im} z|^{-1}\right)+\mathcal{O}\left(\hbar^{-1} \kappa\right) .
\end{aligned}
$$

We then get from (6.7) and (6.9):

$$
\chi_{1}\left(\hbar^{1 / 2} x\right) g\left(\overline{H^{\prime}}\right)=\mathcal{O}\left(\hbar^{1 / 2}\right) .
$$

Notice that we were able to use $\int|\operatorname{Im} z|^{-4} d \tilde{g}<C$, uniformly in $\hbar$, since the size of the support of the function $g$ is independent of $\hbar$. From (6.10), it follows that

$$
\mathcal{U}^{-1} \chi_{1}\left(\hbar^{1 / 2} x\right) g\left(\overline{H^{\prime}}\right) \mathcal{U}=\chi_{1} \mathcal{U}^{-1} g\left(\overline{H^{\prime}}\right) \mathcal{U}=\chi_{1} g(\bar{H} / \hbar)=\mathcal{O}\left(\hbar^{1 / 2}\right),
$$

and (ii) of Proposition 6.2 is proved by multiplying the last equation by $E_{\hbar \Delta_{1}}(\bar{H})$.

\subsection{Local decay in the quasiclassical case.}

Theorem 6.3. Suppose that the Mourre estimate (6.1) holds, and that Conditions (ii) and (T1) of Paragraph 2.3.2 are satisfied. Then there is an interval $\Delta^{\prime} \subset \Delta_{1}$ such that the local decay estimate holds:

$$
\left\|<A>^{-\alpha} e^{-i \bar{H} t / \hbar} g_{\hbar \Delta^{\prime}}(\bar{H}) \bar{P} \phi\right\|_{2} \leq C \hbar^{-N}<t>^{-\alpha}\left\|<A>^{\alpha} \bar{P} \phi\right\|_{2},
$$

where $N$ is an integer depending on $\alpha, n$, and $C$ depends on $\left|\Delta^{\prime}\right|$, but not on $\hbar$.

Proof. Via the unitary transformation $\mathcal{U}$ (introduced in the previous paragraph), (6.12) is equivalent to

$$
\left\|<A>^{-\alpha} e^{-i \overline{H^{\prime}} t} g_{\Delta^{\prime}}\left(\overline{H^{\prime}}\right) \overline{P^{\prime}} \phi\right\|_{2} \leq C \hbar^{-N}<t>^{-\alpha}\left\|<A>^{\alpha} \overline{P^{\prime}} \phi\right\|_{2} .
$$

Notice that $\mathcal{U}$ commutes with $A$. From (6.1), we get by conjugating with $\mathcal{U}$ :

$$
E_{\Delta_{1}}\left(\overline{H^{\prime}}\right) i\left[\overline{H^{\prime}}, A\right] E_{\Delta_{1}}\left(\overline{H^{\prime}}\right) \geq(\theta-\epsilon) E_{\Delta_{1}}\left(\overline{H^{\prime}}\right) .
$$

We have also

$$
\left\|a d_{A}^{(k)}\left(g_{\Delta^{\prime}}\left(\overline{H^{\prime}}\right)\right)\right\| \leq C_{k} \hbar^{-2 k} .
$$

Estimate (6.15) is obtained by using the representation (A.1) and expanding the multicommutator (as in the proof of Lemma A.1, see (A.12)). From (6.14) and (6.15), we get (6.13) following [HSS] and keeping track of the dependence on $\hbar$ in (6.15). 
6.3. Proof of Theorem 2.5. Remarks 2 and 3 after Conditions (T) in Paragraph 2.3.2 show that (A1) and (A2) are satisfied. Clearly, (A3) is also true by (T1) and Condition (i); in fact, $a d_{A}^{(k)}(H)(H+i)^{-1}=\mathcal{O}\left(\hbar^{k}\right)$. (A4) holds modulo the factor $\hbar^{-N}$ (see Theorem 6.3), and (A5) holds modulo $\hbar^{p}$ (see (T4)). Theorem 2.5 follows proceeding as in the proof of Theorem 2.1, and keeping track of $\hbar$.

\section{A. Appendix}

We first present an operator calculus which we then apply to find some norm-estimates on the operator $B$ introduced in Proposition 3.1 that were used at various places in this work. In the subsequent sections, we give the proofs of Propositions 3.1-3.3.

A.1. Operator calculus. The operator calculus presented below is based on a formula due to Helffer and Sjöstrand [HeSj] with estimates of the remainders given in [IS, HS2] (see also [D]). We follow [HS2]. Let $A$ be a self-adjoint operator. For a complex-valued $g \in C_{0}^{\infty}(\mathbb{R})$, we have the representation

$$
g(A)=\int(A-z)^{-1} d \tilde{g}(z),
$$

where the integral is over $\mathbb{C}, \tilde{g}(z)$ is an almost analytic extension of $g$ to the complex plane, and

$$
d \tilde{g}(z) \equiv \frac{1}{2 \pi}\left(\partial_{x}+i \partial_{y}\right) \tilde{g}(z) d x d y
$$

The function $\tilde{g}(z)$ has compact support, and satisfies the estimate

$$
\int|\operatorname{Im}(z)|^{-p} d|\tilde{g}|(z)<\infty, \quad p>0 .
$$

Consequently, the integral (A.1) converges absolutely in norm.

We need also estimates on commutators like $[h(H), f(A)]$, where $H, A$ are selfadjoint operators, $h \in C_{0}^{\infty}$ and $f \in C^{\infty}$. If the multicommutators $a d_{A}^{(k)}(H), k=$ $1, \ldots, n$ are $H$-bounded, and $f$ satisfies the condition given below, then we have the following expansion:

$$
[h(H), f(A)]=\sum_{k=1}^{n-1} \frac{1}{k !} f^{(k)}(A) a d_{A}^{(k)}(h(H))+R_{n},
$$

where

$$
R_{n}=\int(A-z)^{-n} a d_{A}^{(k)}(h(H))(A-z)^{-1} d \tilde{f}(z)
$$

We then have

$$
\left\|R_{n}\right\| \leq C_{n}\left\|a d_{A}^{(n)}(h(H))\right\| \sum_{k=0}^{n+2} \int<x>^{k-n-1}\left|f^{(k)}(x)\right| d x,
$$

where $\langle x\rangle \equiv\left(1+x^{2}\right)^{1 / 2}$. The condition on $f$ is that the integrals in (A.5) exist. For details, see [HS2]. 
A.2. Proof of Proposition 3.1. We want to show that $\left\|\bar{g}_{\Delta^{\prime}}(\bar{H}) \bar{P} g_{\Delta}(H)\right\|<1$ for small $\kappa$, then $B$ is given by the norm-converging Neumann series

$$
B=\left(1-\bar{g}_{\Delta^{\prime}}(\bar{H}) \bar{P} g_{\Delta}(H)\right)^{-1}=\sum_{n=0}^{\infty}\left[\bar{g}_{\Delta^{\prime}}(\bar{H}) \bar{P} g_{\Delta}(H)\right]^{n}
$$

Since $g_{\Delta}(\bar{H})$ commutes with $\bar{P}$, and $\bar{g}_{\Delta^{\prime}} g_{\Delta}=0$, we get $\bar{g}_{\Delta^{\prime}}(\bar{H}) \bar{P} g_{\Delta}(H)=\bar{g}_{\Delta^{\prime}}(\bar{H}) \bar{P}\left(g_{\Delta}(H)-\right.$ $\left.g_{\Delta}(\bar{H})\right)$. Using the second resolvent identity gives

$$
\begin{aligned}
\bar{P}\left(g_{\Delta}(H)-g_{\Delta}(\bar{H})\right) & =-\bar{P} \int\left[(\bar{H}-z)^{-1}-(H-z)^{-1}\right] d \tilde{g}_{\Delta}(z) \\
& =-\int(\bar{H}-z)^{-1} \bar{P} W P(H-z)^{-1} d \tilde{g}_{\Delta}(z) .
\end{aligned}
$$

With $\|W P\| \leq \kappa$, we get

$$
\left\|(\bar{H}-z)^{-1} \bar{P} W P(H-z)^{-1}\right\| \leq \frac{\kappa}{|\operatorname{Im}(z)|^{2}} .
$$

Now $|\operatorname{Im}(z)|^{-2}$ is integrable with respect to $d\left|\tilde{g}_{\Delta}\right|(z)$, see (A.3). The integral in (A.7) is thus bounded by $\kappa C(\Delta)$, where

$$
C(\Delta)=\int|\operatorname{Im}(z)|^{-2} d\left|\tilde{g}_{\Delta}\right|(z)
$$

and this shows existence of $B$ for $\kappa<1 / C(\Delta)$. Moreover

$$
\|B\| \leq \sum_{n=0}^{\infty} \kappa^{n} C(\Delta)^{n}=(1-\kappa C(\Delta))^{-1}
$$

This completes the proof of the proposition.

A.3. Norm estimates on the operator $B$.

Lemma A.1. We have $\forall \phi \in \mathcal{H}$ :

$$
\begin{aligned}
\left\|<A>^{-\alpha} B \phi\right\|_{\mathcal{H}} & \leq C\left\|<A>^{-\alpha} \phi\right\|_{\mathcal{H}}, \\
\left\|<A>^{-\alpha} B^{\prime} \phi\right\|_{\mathcal{H}} & \leq C \kappa\left\|<A>^{-\alpha} \phi\right\|_{\mathcal{H}},
\end{aligned}
$$

where the constants are independent of $\kappa$ for small $\kappa$.

Proof. Due to (A.6) it is enough to show that

$$
\left\|<A>^{-\alpha} \bar{g}_{\Delta^{\prime}}(\bar{H}) \bar{P} g_{\Delta}(H)<A>^{\alpha}\right\| \leq C \kappa .
$$

Due to (A.7) and $\bar{g}_{\Delta^{\prime}}(H) g_{\Delta}(H)=0$, in order to show (A.10), it is enough to show

$$
\left\|\int<A>^{-\alpha}(\bar{H}-z)^{-1} \bar{P} W P(H-z)^{-1} g_{\Delta}(H)<A>^{\alpha} d \tilde{g}_{\Delta^{\prime}}(z)\right\| \leq C \kappa . \text { (A.11) }
$$

We have $\|W P\| \leq \kappa$. Introduce $\left\langle A>^{\alpha}<A>^{-\alpha}\right.$ between $P$ and $(H-z)^{-1}$ in (A.11) and notice that $P<A>^{\alpha}$ is bounded by Condition (A1). The norm of the integrand in (A.11) is then bounded by 


$$
\begin{aligned}
& \kappa|\operatorname{Im}(z)|^{-1}||<A>^{-\alpha}(H-z)^{-1} g_{\Delta}(H)<A>^{\alpha} \| \\
& \leq \kappa|\operatorname{Im}(z)|^{-1}\left(|\operatorname{Im}(z)|^{-1}+\left\|<A>^{-\alpha}\left[(H-z)^{-1} g_{\Delta}(H),<A>^{\alpha}\right]\right\|\right) .
\end{aligned}
$$

To estimate the commutator in the last expression, notice that $\forall z \notin \mathbb{R}, x \in \mathbb{R}, x \mapsto$ $(x-z)^{-1} g_{\Delta}(x) \in C_{0}^{\infty}(\Delta)$. Hence we can apply expansion (A.4)-(A.5) with $h(x) \equiv$ $(x-z)^{-1} g_{\Delta}(x)$, and $f(x)=<x>^{\alpha}$. Estimate (A.5) implies $\left\|R_{n}\right\| \leq C \| a d_{A}^{(n)}((H-$ $\left.z)^{-1} g_{\Delta}(H)\right) \|$. Now

$$
\begin{aligned}
& a d_{A}^{(k)}\left((H-z)^{-1} g_{\Delta}(H)\right) \\
& =\int a d_{A}^{(k)}\left((H-z)^{-1}(H-\zeta)^{-1}\right) d \tilde{g}_{\Delta}(\zeta) \\
& =\sum_{r_{1}+r_{2}=k} C_{r_{1}, r_{2}} \int a d_{A}^{\left(r_{1}\right)}\left((H-z)^{-1}\right) a d_{A}^{\left(r_{2}\right)}\left((H-\zeta)^{-1}\right) d \tilde{g}_{\Delta}(\zeta),
\end{aligned}
$$

for some numbers $C_{r_{1}, r_{2}}$. Therefore

$$
\begin{aligned}
& \left\|a d_{A}^{(k)}\left((H-z)^{-1} g_{\Delta}(H)\right)\right\| \\
& \leq C \sum_{r_{1}+r_{2}=k}\left\|a d_{A}^{\left(r_{1}\right)}\left((H-z)^{-1}\right)\right\| \int\left\|a d_{A}^{\left(r_{2}\right)}\left((H-\zeta)^{-1}\right)\right\| d\left|\tilde{g}_{\Delta}\right|(\zeta)
\end{aligned}
$$

In order to estimate (A.12) further, observe that

$$
\left\|a d_{A}^{(k)}\left((H-z)^{-1}\right)\right\| \leq C \sum_{j=2}^{k+1}|\operatorname{Im}(z)|^{-j}
$$

uniformly in $\kappa$ for small $\kappa$. The integral in (A.12) is thus bounded by

$$
C \int \sum_{j=2}^{k+1}|\operatorname{Im}(\zeta)|^{-j} d\left|\tilde{g}_{\Delta}\right|(\zeta)<\infty
$$

uniformly in $\kappa$, for small $\kappa$. So we get

$$
\left\|a d_{A}^{(k)}\left((H-z)^{-1} g_{\Delta}(H)\right)\right\| \leq C \sum_{j=2}^{k+1}|\operatorname{Im}(z)|^{-j},
$$

and hence the 1.h.s. of (A.12) is indeed bounded by

$$
C \kappa \int\left(|\operatorname{Im}(z)|^{-2}+|\operatorname{Im}(z)|^{-n-2}\right) d\left|\tilde{g}_{\Delta^{\prime}}\right|(z) \leq C \kappa,
$$

uniformly in $\kappa$, for small $\kappa$. This shows (A.8). (A.9) is then readily obtained from the fact that $B^{\prime}=\bar{g}_{\Delta^{\prime}}(\bar{H}) \bar{P} g_{\Delta}(H) B$ and (A.10).

A.4. Proof of Proposition 3.2. For $\phi \in \mathcal{D}\left(<A>^{\alpha}\right), \epsilon \downarrow 0, t \geq 0$ fixed, we show that

$$
\phi_{\epsilon}(t) \equiv<A>^{-\alpha} e^{-i \bar{H} t}(\bar{H}-\omega-i \epsilon)^{-1} P_{d} \phi
$$

is a Cauchy-net in $\mathcal{H}$. We notice that (for $\epsilon>0$ ) 


$$
(\bar{H}-\omega-i \epsilon)^{-1}=i \int_{0}^{\infty} e^{-i(\bar{H}-\omega-i \epsilon) s} d s,
$$

and so we get, using the first resolvent identity:

$$
\begin{aligned}
& (\bar{H}-\omega-i \epsilon)^{-1}-\left(\bar{H}-\omega-i \epsilon^{\prime}\right)^{-1} \\
& =-i\left(\epsilon-\epsilon^{\prime}\right) \int_{0}^{\infty} e^{-i(\bar{H}-\omega-i \epsilon) s} d s \int_{0}^{\infty} e^{-i\left(\bar{H}-\omega-i \epsilon^{\prime}\right) \sigma} d \sigma .
\end{aligned}
$$

Therefore

$$
\begin{aligned}
& \left\|\phi_{\epsilon}(t)-\phi_{\epsilon^{\prime}}(t)\right\|_{\mathcal{H}} \\
& \leq\left|\epsilon-\epsilon^{\prime}\right| \int_{0}^{\infty} e^{-\epsilon s} \int_{0}^{\infty} e^{-\epsilon^{\prime} \sigma}\left\|<A>^{-\alpha} e^{-i \bar{H}(t+s+\sigma)} P_{d} \phi\right\|_{\mathcal{H}} d \sigma d s \\
& \leq\left|\epsilon-\epsilon^{\prime}\right| \int_{0}^{\infty} d s \int_{t+s}^{\infty} d \tau\left\|<A>^{-\alpha} e^{-i \bar{H} \tau} P_{d} \phi\right\|_{\mathcal{H}} \\
& \leq\left|\epsilon-\epsilon^{\prime}\right| \int_{0}^{\infty} d s \int_{s}^{\infty} d \tau(\tau+1)^{-\alpha} C\left\|<A>^{\alpha} \phi\right\|_{\mathcal{H}},
\end{aligned}
$$

where we used the local decay (2.1). Since $\alpha>2$, the double integral in (A.16) is finite, so we get

$$
\left\|\phi_{\epsilon}(t)-\phi_{\epsilon^{\prime}}(t)\right\|_{\mathcal{H}} \leq C\left|\epsilon-\epsilon^{\prime}\right|\left\|<A>^{\alpha} \phi\right\|_{\mathcal{H}}, \quad \forall t \geq 0
$$

which shows that $\phi_{\epsilon}$ is a Cauchy-net, and (i) is proved.

To prove (ii), we remark that from (A.16), we have that the limit $\phi_{0}(t) \equiv \lim _{\epsilon^{\prime} \downarrow 0} \phi_{\epsilon^{\prime}}(t)$ exists, and $\phi_{\epsilon} \rightarrow \phi_{0}$ uniformly in $t$. So $\phi_{0}$ is continuous since the $\phi_{\epsilon}$ 's are.

To prove (iii), remark that $(t \geq 0, \epsilon>0)$ :

$$
e^{-i \bar{H} t}(\bar{H}-\omega-i \epsilon)^{-1}=i e^{-i \omega t} e^{\epsilon t} \int_{t}^{\infty} e^{-i(\bar{H}-\omega-i \epsilon) s} d s
$$

so that we get

$$
\begin{aligned}
& \left\|<A>^{-\alpha} e^{-i \bar{H} t}(\bar{H}-\omega-i \epsilon)^{-1} P_{d} \phi\right\|_{\mathcal{H}} \\
& \leq e^{\epsilon t} \int_{t}^{\infty} e^{-\epsilon s}\left\|<A>^{-\alpha} e^{-i \bar{H} s} P_{d} \phi\right\|_{\mathcal{H}} d s \\
& \leq C\left\|<A>^{\alpha} \phi\right\|_{\mathcal{H}} \int_{t}^{\infty}(s+1)^{-\alpha} d s \\
& \leq C(t+1)^{-\alpha+1}\left\|<A>^{\alpha} \phi\right\|_{\mathcal{H}} .
\end{aligned}
$$

This completes the proof of the proposition.

A.5. Proof of Proposition 3.3. The definition of $\lambda$ is (see the sentence after (3.13))

$$
\lambda=E_{0}+P W P+P W B^{\prime} P-P W\left(\bar{H}-E_{0}-i 0\right)^{-1} P_{d} W P .
$$

We analyze first $P W B^{\prime} P$. Since $B^{\prime}=\bar{g}_{\Delta^{\prime}}(\bar{H}) \bar{P} g_{\Delta}(H)+\mathcal{O}\left(\kappa^{2}\right)$, we have

$$
P W B^{\prime} P=P W \bar{g}_{\Delta^{\prime}}(\bar{H}) \bar{P} g_{\Delta}(H) P+\mathcal{O}\left(\kappa^{3}\right) .
$$


Using $\bar{g}_{\Delta^{\prime}}(\bar{H}) g_{\Delta}(\bar{H})=0$, we get

$$
\begin{aligned}
\bar{g}_{\Delta^{\prime}}(\bar{H}) \bar{P} g_{\Delta}(H) & =\bar{g}_{\Delta^{\prime}}(\bar{H}) \bar{P}\left(g_{\Delta}(H)-g_{\Delta}(\bar{H})\right) \\
& =-\bar{g}_{\Delta^{\prime}}(\bar{H}) \bar{P} \int(\bar{H}-z)^{-1} W P(H-z)^{-1} d \tilde{g}_{\Delta}(z) .
\end{aligned}
$$

Since $(H-z)^{-1}=\left(H_{0}-z\right)^{-1}\left(1-W(H-z)^{-1}\right)$ and $P W$ and $W P$ are $\mathcal{O}(\kappa)$, we deduce that

$$
\bar{g}_{\Delta^{\prime}}(\bar{H}) \bar{P} g_{\Delta}(H)=-\bar{g}_{\Delta^{\prime}}(\bar{H}) \bar{P} \int(\bar{H}-z)^{-1}\left(E_{0}-z\right)^{-1} d \tilde{g}_{\Delta}(z) W P+\mathcal{O}\left(\kappa^{2}\right)
$$

Now

$$
(\bar{H}-z)^{-1}\left(E_{0}-z\right)^{-1}=-\left(\bar{H}-E_{0}\right)^{-1}\left((\bar{H}-z)^{-1}-\left(E_{0}-z\right)^{-1}\right),
$$

and therefore

$$
\begin{aligned}
\bar{g}_{\Delta^{\prime}}(\bar{H}) \bar{P} g_{\Delta}(H) & =\bar{g}_{\Delta^{\prime}}(\bar{H}) \bar{P}\left(\bar{H}-E_{0}\right)^{-1}\left(g_{\Delta}(\bar{H})-g_{\Delta}\left(E_{0}\right)\right) W P+\mathcal{O}\left(\kappa^{2}\right) \\
& =-\bar{g}_{\Delta^{\prime}}(\bar{H}) \bar{P}\left(\bar{H}-E_{0}\right)^{-1} W P+\mathcal{O}\left(\kappa^{2}\right) .
\end{aligned}
$$

Combining this relation with (A.18), we find

$$
P W B^{\prime} P=-P W \bar{g}_{\Delta^{\prime}}(\bar{H})\left(\bar{H}-E_{0}\right)^{-1} \bar{P} W P+\mathcal{O}\left(\kappa^{3}\right) .
$$

Notice that the term of order two in $\kappa$ of $P W B^{\prime} P$ is self-adjoint.

Let us now examine $P W\left(\bar{H}-E_{0}-i 0\right)^{-1} P_{d} W P$, which will give the non-zero anti-self-adjoint contribution of order two in $\kappa$,

$$
\begin{aligned}
& P W\left(\bar{H}-E_{0}-i 0\right)^{-1} P_{d} W P \\
& =P W\left(\bar{H}-E_{0}-i 0\right)^{-1} \bar{P} W P-P W \bar{g}_{\Delta^{\prime}}(\bar{H})\left(\bar{H}-E_{0}\right)^{-1} \bar{P} W P .
\end{aligned}
$$

Observe that the first term on the r.h.s. exists, because of Proposition 3.2 (and the assumption that $\left\langle A>^{\alpha} W P\right.$ is bounded). The second term exists since $E_{0} \notin \operatorname{supp}\left(\bar{g}_{\Delta^{\prime}}\right)$.

Hence it follows from (A.17), (A.20) and (A.21) that

$$
\lambda=E_{0}+P W P-P W\left(\bar{H}-E_{0}-i 0\right)^{-1} \bar{P} W P+\mathcal{O}\left(\kappa^{3}\right) .
$$

Notice that since $P W\left(\bar{H}-E_{0}-i \epsilon\right)^{-1} \bar{P} W P$ converges strongly as $\epsilon \downarrow 0$, then so does its adjoint $P W\left(\bar{H}-E_{0}+i \epsilon\right)^{-1} \bar{P} W P$. The proof is now complete if one observes that the principal value and the delta-function have the representations:

$$
\text { P.V. }\left(\bar{H}-E_{0}\right)^{-1}=\frac{1}{2} \lim _{\epsilon \downarrow 0}\left(\left(\bar{H}-E_{0}-i \epsilon\right)^{-1}+\left(\bar{H}-E_{0}+i \epsilon\right)^{-1}\right)
$$

and

$$
\delta\left(\bar{H}-E_{0}\right)=\frac{1}{2 \pi i} \lim _{\epsilon \downarrow 0}\left(\left(\bar{H}-E_{0}-i \epsilon\right)^{-1}-\left(\bar{H}-E_{0}+i \epsilon\right)^{-1}\right) .
$$

Remark. To show $\|\Gamma\| \leq C \kappa^{2}$ (see (2.3)), notice that $\left\|P W\left(\bar{H}-E_{0} \pm i 0\right)^{-1} P_{d} W P\right\|=$ $\mathcal{O}\left(\kappa^{2}\right)$. This is shown to hold using Proposition 3.2(ii) with $t=0$, and the assumption $P W<A>^{\alpha}=\mathcal{O}(\kappa)$.

Acknowledgement. The authors are grateful to J. Fröhlich, W. Hunziker, A. Soffer and M. Weinstein for useful discussions and to A. Soffer and M. Weinstein for making their work available to us prior to publication. It is a pleasure to thank the referee for useful remarks. 


\section{References}

[AC] Aguilar, J., and Combes, J.M.: A class of analytic perturbations for one-body Schrödinger Hamiltonians. Commun. Math. Phys. 22, 269-279 (1971)

[AHS] Agmon, S., Herbst, I., and Skibsted, E.: Perturbation of embedded eigenvalues in the generalized $N$-body problem. Commun. Math. Phys. 122, 411-438 (1989)

[BC] Balslev, E., and Combes, J.M.: Spectral properties of many-body Schrödinger operators with dilation analytic interactions. Commun. Math. Phys. 22, 280-294 (1971)

[BFS1] Bach, V., Fröhlich, J., and Sigal, I.M.: Mathematical theory of non-relativistic matter and radiation. Lett. in Math. Phys. 34, 183-201 (1995)

[BFS2] Bach, V., Fröhlich, J., and Sigal, I.M.: Quantum Electrodynamics of Confined Nonrelativistic Particles. To appear in Advances in Mathematics

[BFS3] Bach, V., Fröhlich, J., and Sigal, I.M.: Renormalization group Analysis of Spectral Problems in QFT. To appear in Advances in Mathematics

[BP] Buslaev, V., and Perelman, G.: On the stability of solitary waves for nonlinear Schrödinger equations. Am. Math. Soc. Transl. (2) 164, 75-98 (1995)

[BW] Breit, W., and Wigner, E.: Capture of slow neutrons. Phys. Rev. 49, 519-531 (1936)

[CFKS] Cycon, H.L., Froese, R.G., Kirsch, W., and Simon, B.: Schrödinger Operators. Texts and Monographs in Physics, Berlin: Springer-Verlag, 1987

[D] Davies, E.B.: Spectral thoery of differential operators. Cambridge: Cambridge University Press, 1995

[G] Gérard, C.: Resonance theory for periodic Schrödinger operators. Bull. Soc. Math. Fr. 118, 27-54 (1990)

[Gr] Graf, G.M.: The Mourre estimate in the semiclassical limit. Lett. in Math. Phys. 20, 47-54 (1990)

[GS] Gérard, C., and Sigal, I.M.: Space-time picture of semiclassical resonances. Commun. Math. Phys. 145, 281-328 (1992)

[HM] Helffer, B., and Martinez, A.: Comparaison entre les diverses notions de résonances. Preprint (1987)

[HN] Hislop, P.D., and Nakamura, S.: Semiclassical resolvent estimates. Ann. Inst. H. Poincaré A41 (1989)

[HeSj] Helffer, B., and Sjöstrand, J.: Equation de Schrödinger avec champ magnétique et équation de Harper. In: Schrödinger operators, H. Holden, A. Jensen eds., Lecture notes in Physics 345, BerlinHeidelberg-New York: Springer-Verlag, 1989

[HiSig] Hislop, P.D., and Sigal, I.M.: Introduction to spectral theory. Applied Mathematical Sciences 133, NY: Springer, 1996

[Hu1] Hunziker, W.: Distortion analyticity and molecular resonance curves. Ann. Inst. Henri Poincaré 45, 339-358 (1986)

[Hu2] Hunziker, W.: Resonances, metastable states, and exponential decay laws in perturbation theory. Commun. Math. Phys. 132, 177-188 (1990)

[HS1] Hunziker, W., and Sigal, I.M.: The general theory of $N$-body quantum systems. CRM Proceedings and lecture notes 8, 35-72 (1995)

[HS2] Hunziker, W., and Sigal, I.M.: Time-dependent scattering theory of $N$-body quantum systems. Preprint (1997)

[HSS] Hunziker, W., Sigal, I.M., and Soffer, A.: Minimal escape velocities. Preprint (1997)

[IS] Ivrii, V., and Sigal, I.M.: Asymptotics of the ground state energies of large Coulomb systems. Ann. Math. 138, 243-335 (1993)

[KP] Kapur, P.L., and Peierls, R.: The dispersion formula for nuclear reactions. Proc. Roy. Soc. (London) A166, 277-295 (1938)

[LL] Landau, L.D., and Lifshitz, E.M.: Quantum Mechanics. Oxford: Pergamon Press, 1977

[Ort] Orth,A.: Quantum mechanical resonance and binding absorption: The many-body problem. Commun. Math. Phys. 126, 559-573 (1990)

[PF] Pfeifer, P., and Fröhlich, J.: Generalized time-energy uncertainty relations and bounds on life-times of resonances. Rev. Mod. Phys. 67, 759-779 (1995)

[PW] Pillet, C.A., and Wayne, C.E.: Invariant manifolds for a class of dispersive, Hamiltonian, partial differential equations. Preprint (1997)

[S] Schwinger, J.: Field theory of unstable particles. Ann. Phys. 9, 169-193 (1960)

[Sig1] Sigal, I.M.: Complex transformation method and resonances in one-body quantum systems. Ann. Inst. Henri Poincaré 41, 103-114 (1984)

[Sig2] Sigal, I.M.: Non-linear wave and Schrödinger equations. Commun. Math. Phys. 153, 297-320 (1993)

[Sig3] Sigal, I.M.: Scattering Theory for Many-Body Quantum Mechanical Systems. Lecture Notes in Math. 1011, Berlin-Heidelberg-New York: Springer-Verlag, 1983 
[Sim1] Simon, B.: Resonances in $n$-body quantum systems with dilation analytic potentials and the foundations of time-dependent perturbation theory. Ann. Math. 97, 247-274 (1973)

[Sim2] Simon, B.: Resonances and complex scaling: A rigoros overview. Int. J. Quant. Chem. 14, 529-542 (1978)

[Sk1] Skibsted, E.: Truncated Gamow functions and the exponential decay law. Ann. Inst. H. Poincaré 46, 131-153 (1987)

[Sk2] Skibsted, E.: On the evolution of resonance states. J. Math. Anal. Appl. 141, 27-48 (1989)

[SW1] Soffer, A., and Weinstein, M.I.: Multichannel nonlinear scattering for non-integrable equations. Commun. Math. Phys 133, 119-146 (1990)

[SW2] Soffer, A., and Weinstein, M.I.: Multichannel nonlinear scattering, II. The case of anisotropic potentials and data. J. Diff. Eqns. 98, 376-390 (1992)

[SW3] Soffer, A., and Weinstein, M.I.: Time dependent resonance theory. GAFA, to appear

[WW] Weisskopf, V., and Wigner, E.: Berechnung der natürlichen Linienbreite auf Grund der Diracschen Lichttheorie. Z. Phys. 63, 54-73 (1930)

Communicated by B. Simon

This article was processed by the author using the $\mathrm{LTT}_{\mathrm{E}} \mathrm{X}$ style file cljourl from Springer-Verlag 